\title{
Molecular Recording of Mammalian Embryogenesis
}

\section{Citation}

Chan, Michelle M., Zachary D. Smith, Stefanie Grosswendt, Helene Kretzmer, Thomas Norman, Britt Adamson, Marco Jost, Jeffrey J. Quinn, Dian Yang, Alexander Meissner, Jonathan S. Weissman. 2019. Molecular Recording of Mammalian Embryogenesis. Nature 570, no. 7759: 77-82.

\section{Published Version}

doi: 10.1038/s41586-019-1184-5

\section{Permanent link}

http://nrs.harvard.edu/urn-3:HUL.InstRepos:41999978

\section{Terms of Use}

This article was downloaded from Harvard University's DASH repository, and is made available under the terms and conditions applicable to Other Posted Material, as set forth at http:// nrs.harvard.edu/urn-3:HUL.InstRepos:dash.current.terms-of-use\#LAA

\section{Share Your Story}

The Harvard community has made this article openly available.

Please share how this access benefits you. Submit a story.

\section{Accessibility}


2 Michelle M. Chan ${ }^{1,2 *}$, Zachary D. Smith ${ }^{4,5,6 *}$, Stefanie Grosswendt ${ }^{7}$, Helene Kretzmer ${ }^{7}$, Thomas

3 Norman $^{1,2}$, Britt Adamson ${ }^{1,2}$, Marco Jost ${ }^{1,2,3}$, Jeffrey J. Quinn ${ }^{1,2}$, Dian Yang ${ }^{1,2}$, Matthew G.

4 Jones $^{1,2,8}$, Alex Khodaverdian ${ }^{9,10}$, Nir Yosef ${ }^{9,10,11,12}$, Alexander Meissner ${ }^{4,5,7}$, Jonathan S.

5 Weissman $^{1,2}$

6

$7{ }^{1}$ Department of Cellular and Molecular Pharmacology, University of California, San Francisco,

8 San Francisco, CA, USA

$9 \quad{ }^{2}$ Howard Hughes Medical Institute, University of California, San Francisco, San Francisco, CA, 10 USA

$11{ }^{3}$ Department of Microbiology and Immunology, University of California, San Francisco, San

12 Francisco, CA, USA

$13 \quad{ }^{4}$ Broad Institute of MIT and Harvard, Cambridge, Massachusetts, USA

$14{ }^{5}$ Department of Stem Cell and Regenerative Biology, Harvard University, Cambridge,

15 Massachusetts, USA

$16{ }^{6}$ Department of Molecular and Cellular Biology, Harvard University, Cambridge, Massachusetts, 17 USA

$18{ }^{7}$ Department of Genome Regulation, Max Planck Institute for Molecular Genetics, Berlin 14195,

19 Germany

$20{ }^{8}$ Integrative Program in Quantitative Biology, University of California, San Francisco, San

21 Francisco, California, USA

$22{ }^{9}$ Department of Electrical Engineering and Computer Science, University of California,

23 Berkeley, Berkeley, California, USA 
$24{ }^{10}$ Center for Computational Biology, Berkeley, California, USA

$25 \quad{ }^{11}$ Chan Zuckerberg Biohub, San Francisco, California, USA

$26 \quad{ }^{12}$ Ragon Institute of Massachusetts General Hospital, MIT and Harvard, Cambridge,

27 Massachusetts, USA

28

$29 *$ These authors contributed equally to this work

30 Correspondence: jonathan.weissman@ucsf.edu (J.S.W.), meissner@molgen.mpg.de (A.M.)

31 
32 Ontogeny describes the emergence of complex multicellular organisms from single

33 totipotent cells. In mammals, this field is particularly challenging due to the indeterminate

34 relationship between self-renewal and differentiation, variation of progenitor field sizes,

35 and internal gestation. Here, we present a flexible, high information, multi-channel

36 molecular recorder with a single cell (sc) readout and apply it as an evolving lineage tracer

37 to define a mouse cell fate map from fertilization through gastrulation. By combining

38 lineage information with scRNA-seq profiles, we recapitulate canonical developmental

39 relationships between different tissue types and reveal the nearly complete transcriptional

40 convergence of endodermal cells from extra-embryonic and embryonic origins. Finally, we

41 apply our cell fate map to estimate the number of embryonic progenitor cells and their

42 degree of asymmetric partitioning during specification. Our approach enables massively

43 parallel, high-resolution recording of lineage and other information in mammalian systems

44 to facilitate a quantitative framework for understanding developmental processes. 
Development of a multicellular organism from a single cell is an astonishing process.

47 Classic lineage tracing experiments using C.elegans revealed surprising outcomes, including

48 deviations between lineage and functional phenotype, but nonetheless benefited from the highly

49 deterministic nature of this organism's development ${ }^{1}$. Alternatively, more complex species

50 generate larger, more elaborate structures that progress through multiple transitions, raising

51 questions regarding the coordination between specification and commitment to ensure faithful

52 recapitulation of an exact body plan $^{2,3}$. Single cell RNA-sequencing (scRNA-seq) has permitted

53 unprecedented explorations into cell type heterogeneity, producing profiles of developing

54 flatworms $^{4,5}$, frogs $^{6}$, zebrafish $^{7,8}$, and mice ${ }^{9,10}$. More recently, CRISPR-Cas9-based technologies

55 have been applied to record cell lineage ${ }^{11-13}$, and combined with scRNA-seq to generate fate

56 maps in zebrafish ${ }^{14-16}$. However, these technologies include only one or two bursts of barcode

57 diversity generation, which may be limiting for other applications or organisms.

58 An ideal molecular recorder for these questions would possess the following

59 characteristics: 1) minimal impact on cellular phenotype; 2) high information content to account

60 for hundreds of thousands of cells; 3) a single cell readout for simultaneous profiling of

61 functional state ${ }^{14-16}$; 4) flexible recording rates that can be tuned to a broad temporal range; and

62 5) continuous generation of diversity throughout the experiment. The last point is especially

63 relevant for mammalian development, where spatial plans are gradually and continuously

64 specified and may originate from small, transient progenitor fields. Moreover, scRNA-seq has

65 revealed populations of cells with a continuous spectrum of phenotypes, implying that

66 differentiation does not occur instantaneously, further motivating the need for an evolving

67 recorder ${ }^{17}$. 
69 lineage history in mice. Our CRISPR-Cas9-based recorder is capable of high information content

70 and multi-channel recording with readily tunable mutation rates. We employ the recorder as a

71 continuously evolving lineage tracer to observe the fate map underlying embryogenesis through

72 gastrulation, recapitulating canonical paradigms and illustrating how lineage information may

73 facilitate the identification of novel cell types.

74

75 
Results

\section{A transcribed, multi-channel, and continuously evolving molecular recorder}

To achieve our goal of a tunable, high information content molecular recorder, we

utilized Cas9 to generate insertions or deletions (indels) upon repair of double-stranded breaks, which are inherited in the next generation of cells ${ }^{11-16}$. We record within a 205 base pair,

81 synthetic DNA "target site" containing three "cut sites" and a static 8 base pair "integration

82 barcode" (intBC), which are delivered in multiple copies via piggyBac transposition (Fig. 1a, b).

83 We embedded this sequence into the 3'UTR of a constitutively transcribed fluorescent protein to

84 enable profiling from the transcriptome. A second cassette encodes three independently

85 transcribed and complementary guide RNAs to permit recording of multiple, distinct signals

86 (Fig. 1a, b) $)^{18}$.

87 Our system is capable of high information storage due to the diversity of heritable repair

88 outcomes, and the large number of targeted sites, which can be distinguished by the intBC (Fig.

89 1c). DNA repair generates hundreds of unique indels, and the distribution for each cut site is

90 different and nonuniform: some produce highly biased outcomes while others create a diverse

91 series (Fig. 1c, Extended Data Fig. 1) ${ }^{19-21}$. To identify sequences that can tune the mutation rate

92 of our recorder for timescales that are not pre-defined, and may extend from days to months, we

93 screened several guide RNA series containing mismatches to their targets ${ }^{22}$ by monitoring their

94 activity on a GFP reporter over a 20-day timecourse and selected those that demonstrated a broad

95 dynamic range (Fig. 1d). Slower cutting rates may improve viability in vivo, as frequent Cas9-

96 mediated double-strand breaks can cause cellular toxicity ${ }^{23,24}$. To demonstrate information

97 recovery from single cell transcriptomes, we stably transduced K562 cells with our technology

98 and generated a primary, cell-barcoded cDNA pool via the 10x Genomics platform, allowing us 
99 to assess global transcriptomes and specifically amplify mutated target sites (Extended Data 100 Fig. 1c).

101

102 Tracing cell lineages in mouse development

103 We next applied our technology to map cell fates during mouse early development from

104 totipotency onwards. We integrated multiple target sites into the genome, delivered constitutive

105 Cas9-GFP encoding sperm into oocytes to initiate cutting, and isolated embryos for analysis at

$106 \sim$ embryonic day (E)8.5 or E9.5 (Fig. 2a, Methods). To confirm our lineage tracing capability,

107 we amplified the target site from bulk placenta, yolk sac, and three embryonic fractions from an

108 E9.5 embryo and recapitulated their expected relationships using the similarity of their indel

109 proportions (Fig. 2b, Extended Data Figure 2).

110 Following this in vivo proof of principle, we generated single cell data from additional

111 embryos (Extended Data Figure 3). We collected scRNA-seq data for 7,364 - 12,990 cells

112 from 7 embryos $(\sim 15.8 \%-61.4 \%$ of the total cell count $)$ and recovered $167-2,461$ unique

113 lineage identities $(\geq 1$ target site recovered for $15 \%-75 \%$ of cells from 3 to 15 intBCs, Fig. 2c,

114 Extended Data Figure 4). Many target sites are either lowly or heterogeneously represented,

115 which we improved by changing the promoter from a truncated form of Efl $\alpha$ to an intron-

116 containing version (see embryo 7, Extended Data Figure 4) ${ }^{25}$.

117 We estimated the indel likelihood distribution by combining data from all seven embryos.

118 Many indels are shared with K562 cells, though their likelihoods differ, suggesting that cell type

119 or developmental status may influence repair outcomes (Fig. 2d, Extended Data Figure 1, 4f $)^{19}$.

120 Our ability to independently measure and control the rate of cutting across the target site is

121 preserved in vivo, with minimal interference between cut sites except when using combinations 
122 of the fastest guides that may lead to end-joining between simultaneous double strand breaks

123 (Fig. 2e). The fastest cutters result in higher proportions of cells with identical indels, indicating

124 earlier mutations in development, which correspondingly reduce indel diversity (Fig. 2f, g).

125 Importantly, the lineage tracer retains additional recording capacity beyond the temporal interval 126 studied here, as most embryos still have unmodified cut sites (Fig. 2f).

128 Assigning cellular states by simultaneous scRNA-seq

129 Next, to ascertain cell function, we utilized annotations from a compendium of wild-type

130 mouse gastrulation (E6.5 - E8.5). We assigned cells from lineage-traced embryos by their

131 proximity to each cell state expression signature and aged each embryo by their tissue

132 proportions compared to each stage (Fig. 3a-c) ${ }^{26}$. We proceeded with six of our seven embryos,

133 as they appeared to be morphologically normal and included every expected tissue type: two

134 mapped most closely to E8.5, and the remaining four mapped to E8.0 (Extended Data Fig. 5).

135 Placenta was not specifically isolated, but is present in four of six embryos, serving as a valuable 136 outgroup to establish our ability to track transitions to the earliest bifurcation.

137 We also developed breeder mice that would enable facile exploration of all stages of 138 development by injecting target sites into Cas9 negative backgrounds. This approach

139 substantially increases the number of stably integrated target sites ( 20$)$. Resulting mice can be 140 crossed with Cas9 expressing strains to yield viable Cas $9^{+} \mathrm{F} 1$ litters that maintain continuous,

141 stochastic indel generation into adulthood, demonstrating that cutting does not noticeably

142 interfere with normal animal development (Extended Data Fig 6).

\section{Single cell lineage reconstruction of mouse embryogenesis}


We developed phylogenetic reconstruction strategies to specifically exploit the

145 characteristics of our lineage tracer, namely categorical indels, irreversibility of mutations, and

146 presence of missing values (Extended Data Figure 7, Methods). We determined the best

147 reconstruction by summing the log-likelihoods for all indels that appear in the tree using

148 likelihoods estimated from embryo data (Extended Data Figures 4 and 7). When cell type

149 identity from scRNA-seq is overlaid onto the tree, we observe functional restriction during

150 development, with fewer cell types represented as we move from root to leaves (Fig. 4a, b,

151 Extended Data Figure 8).

152 scRNA-seq-based strategies for ordering cells, such as trajectory inference, typically

153 assume that functional similarity reflects close lineage ${ }^{17}$. To investigate this question directly,

154 we used a modified Hamming distance to measure pairwise lineage distance and compared them

155 to RNA-seq correlation. Generally, cells separated by a smaller lineage distance have more

156 similar transcriptional profiles, though this relationship is clearer for some embryos than others

157 (Fig. 4c, Extended Data Figure 9). This result is consistent with the notion of continuous

158 restriction of potency as cells differentiate into progressively differentiated types.

159 We also developed a shared progenitor score that estimates the degree of common

160 ancestry between different tissues by evaluating the number and specificity of shared nodes in

161 the tree (Methods). Despite the stochastic timing of indel formation, this approach can

162 reproducibly recover emergent tissue relationships, such as possible shared origins between

163 anterior somites and paraxial mesoderm or neuromesodermal progenitors and the future spinal

164 cord (Fig. 4d). The full map of shared progenitor scores can be clustered to create a

165 comprehensive picture of tissue relationships during development (Extended Data Fig. 8d). 
While our reconstructed tissue relationships generally recapitulate canonical knowledge, extra-embryonic and embryonic endoderm display consistent and unexpectedly close ancestry

170 despite their independent origins from the hypoblast and embryo-restricted epiblast (Fig. 5a,

171 Extended Data Figure 9). Manual inspection of the trees revealed a subpopulation of cells that

172 appear transcriptionally as embryonic endoderm but that lineage analysis places within extra-

173 embryonic branches (Fig 4c, blue). Consistent with this finding, an earlier, targeted study using

174 marker-directed lineage tracing identified latent extra-embryonic contribution to the developing

175 hindgut during gastrulation, although it was not possible to broadly evaluate their

176 transcriptomes ${ }^{27}$.

Here, scRNA-seq profiles collected in tandem with the lineage readout allow us to assess

178 the degree of convergence towards a functional endoderm signature and identify distinguishing

179 genes. Endoderm-classified cells derived from extra-embryonic origin are most similar to the

180 endoderm cell type, but do share slightly higher similarity with yolk sac that is not apparent

181 within the t-sne projection of the full embryo (Fig. 5b, Extended Data Figure 10). Given these

182 independent origins, we might expect a subtle, but persistent, transcriptional signature reflecting

183 their developmental history. Strikingly, when we separate endoderm cells according to their

184 lineage, we identify two X-linked genes, Trap1a and Rhox5, general markers for extra-

185 embryonic tissue ${ }^{28,29}$ that are consistently upregulated in the extra-embryonic origin endoderm

186 across embryos (K-S test, Bonferroni corrected $P$-value $<0.05$, Fig. 5d, e). Notably, in other

187 RNA-seq studies, these relationships are not captured by whole embryo clustering, and are only

188 found by specific examination of the hindgut (Extended Data Figure 10) ${ }^{9,30}$. These 
189 observations confirm that our lineage tracer can successfully pinpoint instances of convergent

190 transcriptional regulation.

\section{Towards a quantitative fate map}

193 Simultaneous single cell lineage tracing with phenotype provides the unique opportunity

194 to infer the cellular potency and specification biases of ancestral cells as reconstructed by our

195 fate $\mathrm{map}^{31,32}$. Each node within the tree represents a unique lineage identity stemming from a

196 single reconstructed progenitor cell, allowing us to estimate lower boundaries of their field size

197 (Methods). We investigated the founding number of progenitors during the earliest transitions in

198 cellular potential. We defined totipotency as a node that gives rise to both embryonic and extra-

199 embryonic ectodermal/placental cell types and tiered pluripotency into "early" and "late"

200 according to the presence of extra-embryonic endoderm (Fig. 6a) ${ }^{33}$. The contributions of these

201 founders to extant lineages are asymmetric, suggesting that even though a progenitor may be

202 biased towards a specific fate, it retains the ability to generate other cell types. Lower bound

203 estimates from our data suggest a range of 1-6 totipotent cells, 10-20 early, and 18-51 late

204 pluripotent progenitors (Fig. 6b). The variable number of multipotent cells at these stages may

205 reflect an encoded robustness that ensures successful assembly of the functioning organism,

206 particularly given that a single pluripotent cell can generate all somatic lineages in an embryo ${ }^{34}$.

207 Future studies using more replicates generated by breeding may enable statistical approaches to 208 evaluate these organism-scale developmental considerations.

\section{Discussion}


In this study, we present cell fate maps underlying mammalian gastrulation using a

212 technology for high information and continuous recording. Several key ideas have emerged,

213 including the transformative nature of CRISPR-Cas9-directed mutation with a single cell RNA-

214 seq readout ${ }^{14-16}$, how information about a cell's history recorded by this technology can

215 complement RNA-seq profiles to characterize cell type, and an early framework for

216 quantitatively understanding stochastic transitions during mammalian development.

217 The modularity of our recorder allows for substitutions that will increase its breadth of

218 applications. Here, we use three constitutively expressed guide RNAs to record continuously

219 over time, but future modifications could employ environmentally-responsive promoters that

220 sense stress, neuronal action potentials, or cell-to-cell contacts ${ }^{35}$, or combine these approaches

221 for multifactorial recording. Similarly, Cas9-derived base editors ${ }^{36}$, including those that create

222 diverse mutations ${ }^{37}$ could allow for content-recording in cells that are particularly sensitive to

223 nuclease-directed DNA double strand breaks ${ }^{23,24}$.

224 Our cell fate map identifies phenotypic convergence of independent cell lineages,

225 showcasing the power of unbiased organism-wide lineage tracing to separate populations that

226 appear similar in scRNA-seq alone. Specifically, we substantiate the extra-embryonic origin of a

227 subset of cells that resemble embryonic endoderm. While the initial specification of these

228 lineages are known to rely on redundant regulatory programs, they are temporally separated by

229 several days, emerge from transcriptionally and epigenetically distinct progenitors, and form

230 terminal cell types with highly divergent functions. The identification of highly predictive

231 markers that segregate by origin, such as Trap1a, provides a clear outline for further exploration

232 through spatial transcriptomics ${ }^{38,39,40}$. More generally, our approach can be used to investigate

233 other convergent processes or to discriminate heterogeneous cell states that represent persistent 
234 signatures of a cell's past, which will be critical for the assembly of a comprehensive cell atlas ${ }^{41}$.

235 The scope of transdifferentiation within mammalian ontogenesis remains largely unexplored, but 236 can be practically inventoried using our system.

237 Ultimately, our technology is designed to quantitatively address previously opaque 238 questions in ontogenesis. Higher order issues of organismal regulation, such as the location, 239 timing, and stringency of developmental bottlenecks, as well as the corresponding likelihoods of 240 state transitions to different cellular phenotypes, can be modeled from the assembly of historical 241 relationships. Our hope is that characterization of these attributes will lead to new insights that 242 connect large-scale developmental phenomena to the molecular regulation of cell fate decision243 making. 


\section{References}

246 1. Sulston, J. E., Schierenberg, E., White, J. G. \& Thomson, J. N. The embryonic cell lineage 247 of the nematode Caenorhabditis elegans. Developmental Biology 100, 64-119 (1983).

248 2. Pijuan-Sala, B., Guibentif, C. \& Göttgens, B. Single-cell transcriptional profiling: a 249 window into embryonic cell-type specification. Nat. Rev. Mol. Cell Biol. 19, 399-412 $250 \quad$ (2018).

251 3. Zernicka-Goetz, M. Patterning of the embryo: the first spatial decisions in the life of a 252 mouse. Development 129, 815-829 (2002).

253 4. Fincher, C. T., Wurtzel, O., de Hoog, T., Kravarik, K. M. \& Reddien, P. W. Cell type 254 transcriptome atlas for the planarian Schmidtea mediterranea. Science 360, eaaq1736 $255 \quad$ (2018).

256 5. Plass, M. et al. Cell type atlas and lineage tree of a whole complex animal by single-cell 257 transcriptomics. Science 360, eaaq1723 (2018).

258 6. Briggs, J. A. et al. The dynamics of gene expression in vertebrate embryogenesis at 259 single-cell resolution. Science 360, eaar5780 (2018).

2607 Farrell, J. A. et al. Single-cell reconstruction of developmental trajectories during 261 zebrafish embryogenesis. Science 360, eaar3131 (2018).

262 8. Wagner, D. E. et al. Single-cell mapping of gene expression landscapes and lineage in the 263 zebrafish embryo. Science 360, 981-987 (2018).

264 9. Ibarra-Soria, X. et al. Defining murine organogenesis at single-cell resolution reveals a 265 role for the leukotriene pathway in regulating blood progenitor formation. Nat. Cell Biol.

$266 \quad$ 20, 127-134 (2018).

267 10. Han, X. et al. Mapping the Mouse Cell Atlas by Microwell-Seq. Cell 172, 1091-1107.e17 
269 11. Perli, S. D., Cui, C. H. \& Lu, T. K. Continuous genetic recording with self-targeting CRISPR-Cas in human cells. Science 353, aag0511-aag0511 (2016).

271 12. Kalhor, R. et al. Developmental barcoding of whole mouse via homing CRISPR. Science 361, eaat9804 (2018).

273 13. Frieda, K. L. et al. Synthetic recording and in situ readout of lineage information in single cells. Nature 541, 107-111 (2017).

275 14. Raj, B. et al. Simultaneous single-cell profiling of lineages and cell types in the vertebrate brain. Nat. Biotechnol.36, 442-450 (2018).

277 15. Alemany, A., Florescu, M., Baron, C. S., Peterson-Maduro, J. \& van Oudenaarden, A.

278 Whole-organism clone tracing using single-cell sequencing. Nature 556, 108-112 (2018).

279 16. Spanjaard, B. et al. Simultaneous lineage tracing and cell-type identification using CRISPR-Cas9-induced genetic scars. Nat. Biotechnol. 36, 469-473 (2018).

281 17. Tanay, A. \& Regev, A. Scaling single-cell genomics from phenomenology to mechanism. Nature 541, 331-338 (2017).

283 18. Adamson, B. et al. A Multiplexed Single-Cell CRISPR Screening Platform Enables Systematic Dissection of the Unfolded Protein Response. Cell 167, 1867-1882.e21 (2016).

19. van Overbeek, M. et al. DNA Repair Profiling Reveals Nonrandom Outcomes at Cas9Mediated Breaks. Mol. Cell 63, 633-646 (2016).

288 20. Schimmel, J., Kool, H., van Schendel, R. \& Tijsterman, M. Mutational signatures of non289 homologous and polymerase theta-mediated end-joining in embryonic stem cells. EMBO J. 36, 3634-3649 (2017). 
291 21. Lemos, B. R. et al. CRISPR/Cas9 cleavages in budding yeast reveal templated insertions 292 and strand-specific insertion/deletion profiles. Proc. Natl. Acad. Sci. U.S.A. 115, E2040$293 \quad$ E2047 (2018).

294 22. Gilbert, L. A. et al. Genome-Scale CRISPR-Mediated Control of Gene Repression and $295 \quad$ Activation. Cell 159, 647-661 (2014).

296 23. Ihry, R. J. et al.p53 inhibits CRISPR-Cas9 engineering in human pluripotent stem cells. $297 \quad$ Nat.Med.337, 816 (2018).

298 24. Haapaniemi, E., Botla, S., Persson, J., Schmierer, B. \& Taipale, J. CRISPR-Cas9 genome 299 editing induces a p53-mediated DNA damage response. Nat.Med.19, 1 (2018).

$30025 . \quad$ Kim, S.-Y., Lee, J.-H., Shin, H.-S., Kang, H.-J. \& Kim, Y.-S. The human elongation 301 factor 1 alpha (EF-1 alpha) first intron highly enhances expression of foreign genes from 302 the murine cytomegalovirus promoter. J. Biotechnol. 93, 183-187 (2002).

303 26. Butler, A., Hoffman, P., Smibert, P., Papalexi, E. \& Satija, R. Integrating single-cell 304 transcriptomic data across different conditions, technologies, and species. Nat. Biotechnol. $305 \quad 36,411-420(2018)$.

306 27. Kwon, G. S., Viotti, M. \& Hadjantonakis, A.-K. The endoderm of the mouse embryo 307 arises by dynamic widespread intercalation of embryonic and extraembryonic lineages.

$308 \quad$ Dev. Cell 15, 509-520 (2008).

309 28. Eakin, G. S. \& Hadjantonakis, A.-K. Sex-specific gene expression in preimplantation 310 mouse embryos. 7, 205 (2006).

311 29. Li, C.-S. et al. Trapla is an X-linked and cell-intrinsic regulator of thymocyte 312 development. Cell.Mol.Immunol. 14, 685-692 (2017).

313 30. Pijuan-Sala, B. et al. A single-cell molecular map of mouse gastrulation and early 
organogenesis. Nature 566, 490-495 (2019).

315 31. Soriano, P. \& Jaenisch, R. Retroviruses as probes for mammalian development: allocation 316 of cells to the somatic and germ cell lineages. Cell 46, 19-29 (1986).

317 32. Jaenisch, R. Mammalian neural crest cells participate in normal embryonic development 318 on microinjection into post-implantation mouse embryos. Nature 318, 181-183 (1985).

319 33. Nichols, J. \& Smith, A. Naive and primed pluripotent states. Cell Stem Cell 4, 487-492 $320 \quad$ (2009).

321 34. Wang, Z. \& Jaenisch, R. At most three ES cells contribute to the somatic lineages of 322 chimeric mice and of mice produced by ES-tetraploid complementation. Developmental 323 Biology 275, 192-201 (2004).

324 35. Baeumler, T. A., Ahmed, A. A. \& Fulga, T. A. Engineering Synthetic Signaling Pathways 325 with Programmable dCas9-Based Chimeric Receptors. Cell Rep 20, 2639-2653 (2017).

326 36. Komor, A. C., Kim, Y. B., Packer, M. S., Zuris, J. A. \& Liu, D. R. Programmable editing 327 of a target base in genomic DNA without double-stranded DNA cleavage. Nature 533, $328 \quad 420-424(2016)$.

329 37. Hess, G. T. et al. Directed evolution using dCas9-targeted somatic hypermutation in 330 mammalian cells. Nat. Methods 13, 1036-1042 (2016).

331 38. Hou, J. et al. A systematic screen for genes expressed in definitive endoderm by Serial 332 Analysis of Gene Expression (SAGE). BMC Dev. Biol. 7, 92 (2007).

333 39. Wang, G., Moffitt, J. R. \& Zhuang, X. Multiplexed imaging of high-density libraries of 334 RNAs with MERFISH and expansion microscopy. Sci Rep 8, 4847 (2018).

335 40. Shah, S., Lubeck, E., Zhou, W. \& Cai, L. In Situ Transcription Profiling of Single Cells 336 Reveals Spatial Organization of Cells in the Mouse Hippocampus. Neuron 92, 342-357 
338 41. Regev, A. et al. The Human Cell Atlas. Elife 6, 503 (2017).

339 42. Tzouanacou, E., Wegener, A., Wymeersch, F. J., Wilson, V. \& Nicolas, J.-F. Redefining

340 the progression of lineage segregations during mammalian embryogenesis by clonal analysis. Dev. Cell 17, 365-376 (2009).

\section{Supplementary References}

344 43. Schiml, S., Fauser, F. \& Puchta, H. The CRISPR/Cas system can be used as nuclease for 345 in plantagene targeting and as paired nickases for directed mutagenesis in Arabidopsis resulting in heritable progeny. Plant $J \mathbf{8 0}, 1139-1150$ (2014).

347 44. Ren, X. et al. Performance of the Cas9 Nickase System in Drosophila melanogaster. G3 4, 1955-1962 (2014).

45. Kimura, Y., Hisano, Y., Kawahara, A. \& Higashijima, S.-I. Efficient generation of knockin transgenic zebrafish carrying reporter/driver genes by CRISPR/Cas9-mediated genome engineering. Sci Rep 4, 206-7 (2014).

352 46. Dong, Z., Dong, X., Jia, W., Cao, S. \& Zhao, Q. Improving the efficiency for generation of genome-edited zebrafish by labeling primordial germ cells. International Journal of Biochemistry and Cell Biology 55, 329-334 (2014).

47. Bao, Z. et al. Homology-Integrated CRISPR-Cas (HI-CRISPR) System for One-Step Multigene Disruption in Saccharomyces cerevisiae. ACS Synth. Biol. 4, 585-594 (2014).

357 48. Zhou, H., Liu, B., Weeks, D. P., Spalding, M. H. \& Yang, B. Large chromosomal 358 deletions and heritable small genetic changes induced by CRISPR/Cas9 in rice. Nucleic Acids Research 42, 10903-10914 (2014). 
49. Jiang, W. et al. Demonstration of CRISPR/Cas9/sgRNA-mediated targeted gene modification in Arabidopsis, tobacco, sorghum and rice. Nucleic Acids Research 41, e188-e188 (2013).

363 50. Feng, Z. et al. Efficient genome editing in plants using a CRISPR/Cas system. Nature Publishing Group 23, 1229-1232 (2013).

365 51. DiCarlo, J. E. et al. Genome engineering in Saccharomyces cerevisiae using CRISPR-Cas systems. Nucleic Acids Research 41, 4336-4343 (2013).

367 52. Jacobs, J. Z., Ciccaglione, K. M., Tournier, V. \& Zaratiegui, M. Implementation of the 368 CRISPR-Cas9 system in fission yeast. Nature Communications 5, 1-5 (1AD).

369 53. Wang, H. et al. One-step generation of mice carrying mutations in multiple genes by 370 CRISPR/Cas-mediated genome engineering. Cell 153, 910-918 (2013).

371 54. Platt, R. J. et al. CRISPR-Cas9 knockin mice for genome editing and cancer modeling. Cell 159, 440-455 (2014).

373 55. Yoshida, N. \& Perry, A. C. F. Piezo-actuated mouse intracytoplasmic sperm injection (ICSI). Nat Protoc 2, 296-304 (2007).

375 56. Gusfield, D. Efficient algorithms for inferring evolutionary trees. Networks 21, 19-28 (1991).

377 57. Jahn, K., Kuipers, J. \& Beerenwinkel, N. Tree inference for single-cell data. Genome Biol. 378 17, 86 (2016). 


\section{Acknowledgments}

381 We would like to thank members of the Weissman, Meissner, and Yosef labs, particularly J.

382 Charlton and A. Arczewska for assistance with animal imaging, A. Kumar for explorations of

383 extraembryonic endoderm, and L. Gilbert and M. Horlbeck for guidance on technology design,

384 as well as Eric Chow and Derek Bogdanoff from the UCSF Center for Advanced Technology for

385 sequencing. This work was funded by National Institutes of Health Grants R01 DA036858 and

386 1RM1 HG009490-01 (J.S.W.), P50 HG006193 and R01 HD078679 (A.M.), F32 GM116331

387 (M.J.), and F32 GM125247 (J.J.Q), and Chan-Zuckerberg Initiative 2018-184034. J.S.W. is a

388 Howard Hughes Medical Institute Investigator. M.M.C. is a Gordon and Betty Moore fellow of

389 the Life Sciences Research Foundation. T.M.N. is a fellow of the Damon Runyon Cancer

390 Research Foundation. S.G., H.K., and A.M. are supported by the Max Planck Society.

391 Author Contributions

392 M.M.C., Z.D.S., A.M. and J.S.W. were responsible for the conception, design, and interpretation 393 of the experiments and wrote the manuscript. M.M.C. and Z.D.S. conducted experiments and

394 M.M.C. developed the analysis, with input from Z.D.S. S.G. and H.K. provided annotations for 395 RNA-seq data and assisted in experimental and analytical optimization. B.A., T.M.N, and M.J.

396 provided vectors, experimental protocols, and advice. J.Q. and D.Y. prepared several sequencing

397 libraries and were engaged in discussion. M.G.J, A.K, and N.Y provided phylogenetic

398 reconstruction strategies.

399 Author Information

400 The authors declare no competing interests. Correspondence and request for materials should be 401 addressed to Jonathan Weissman (jonathan.weissman@ucsf.edu) and Alexander Meissner 402 (meissner@molgen.mpg.de). 


\section{Figure Legends}

\section{Figure 1: Optimization of a multi-purpose molecular recorder}

405 a. Target site (top) and three guide (bottom) cassettes. The target site consists of an integration barcode (intBC) and three cut sites for Cas9-based recording. Three different single guide RNAs (sgRNAs) are each controlled by independent promoters (in this study, mU6, hU6, and bU6).

b. Molecular recording principle. Each cell contains multiple genomic, intBCdistinguishable target site integrations. sgRNAs direct Cas9 to cognate cut sites to generate insertion (red) or deletion mutations. Here, Cas9 is either ectopically delivered or induced by doxycycline.

c. Percentage of uniquely marked reads recovered after recording within a K562 line with 10 intBCs for 6 days using the following information: site 1 only with intBCs masked, sites 1-3 (All) with intBCs masked, and sites 1-3 (All) with intBCs considered. Information content scales with number of sites and presence of the intBC.

d. sgRNA mismatches alter mutation rate. Seven protospacers were integrated into the coding sequence of a GFP reporter to infer mutation rate by the fraction of positive cells over a 20 day time course. Single or dual mismatches were made in guides according to proximity to the PAM: region 1 (proximal), region 2, and region 3 (distal). Guides against Gal4-4 and the GFP coding sequence act as negative and positive controls. Bold 
a. Lineage tracing in mouse experiments. The target site (within mCherry's 3'UTR) and the three guide cassettes are encoded into a single piggyBac transposon vector (ITRs, inverted terminal repeats). The vector, transposase mRNA, and Rosa26::Cas9:EGFP sperm are injected into oocytes to ensure early integration and tracing in all subsequent cells after zygotic genome activation. Transferred embryos are then recovered after gastrulation.

b. Pearson correlation coefficient heatmap of indel proportions recovered from bulk tissue of an E9.5 embryo (see also Extended Data Figure 2).

c. Indel frequency distribution estimated from 40 independent target sites from all embryos. Each site produces hundreds of outcomes for high information encoding. See Extended Data Figure 4 and Methods for frequency calculation. The indel code along the x-axis is as follows: "Alignment Coordinate: Indel Size Indel type (Insertion or Deletion)."

d. Proportion of indels that span one, two, or three sites, shown per site. Each dot denotes one of 40 independent intBCs and sums to one across site-spanning indels. Colors indicate the guide array: $\mathrm{P}=$ no mismatches; $1=$ mismatch in region $1 ; 2=$ mismatch in region 2 .

e. Percentage of cells with mutations according to guide complementarity. Indel proportions within one mouse depend on timing: mutations that happen earlier in development are propagated to more cells. Dots represent site 1 measurements from independent intBCs; $\mathrm{N}=4,24$, and 18 for $\mathrm{P}, 2$, and 1 region mismatches.

f. Indel diversity is inversely related to cutting efficiency for site 1 as in e. Early mutations due to fast cutting are propagated to more cells, leading to smaller numbers of unique indels. 


\section{Figure 3: Assigning cellular phenotype by scRNA-seq}

a. Images of a lineage-traced E8.5 embryo (embryo 2 of 7 for which single cell data was collected, see Extended Data Figure 3), including for Cas9:EGFP and the mCherry:target site.

\section{Figure 4: Single cell lineage reconstruction of mouse embryogenesis}

a. Reconstructed lineage tree comprised of 1,732 nodes for embryo 2 with three lineages highlighted. Each branch represents an indel generation event.

b. Example paths from tree in a highlighted by color. Cells for each node in the path are overlaid onto the plot from Figure 3b, with tissue proportions as a pie chart. Tissue representation decreases with increased tree depth, indicating functional restriction. Bifurcating sublineages are included for the top and bottom paths. In the top (red) path, 
this bifurcation occurs within the final branch after primitive blood specification. In the bottom (blue) path, bifurcation happens early within bipotent cells that become either gut or visceral endoderm.

c. Violin plots of the pairwise relationship between lineage and expression for single cells. Lineage distance uses a modified Hamming distance normalized to the number of shared cut sites. Pearson correlation decreases with increasing lineage distance, showing that

\section{embryonic endoderm}

a. Shared progenitor score heatmap for embryo 2 reconstructs expected relationships. The number of nodes that include cells from different lineages is highlighted (Heterogeneous nodes). See Extended Data Figure 9 for additional embryos.

b. For cells from embryo 2, the relative distance from the mean expression profile of either the endoderm or the extra-embryonic endoderm cluster according to origin (Endo or XEndo). 
c. Endoderm cell lineage tree from embryo 2 with expression heatmap for two extraembryonic marker genes. Middle bar indicates lineage: dark blue, extra-embryonic; light blue, embryonic; grey, ambiguous.

d. Expression boxplots for Trap1a and Rhox 5 confirms consistent differential expression across lineage-traced embryos according to their embryonic or extra-embryonic ancestry. Red line highlights median, edges the interquartile range, whiskers the Tukey Fence, and crosses outliers. N's, the number of recovered XEndo origin cells of either embryonic (E) or Extraembryonic (X) function per embryo.

a. Relative tissue distribution of cells descended from reconstructed or profiled pluripotent progenitor cells for embryo 2. "Profiled" is a unique lineage identity of multiple cells Nodes highlighted in grey with asterisk overlasy give rise to primordial germ cells directly observed in the data. Pluripotent cells form all germ layers, but show asymmetric propensities towards different cell fates, possibly reflecting positional biases. (lineages 1, 4, and 5 include 9, 1, and 1 PGCs each). Color assignments as in Figures 3. b. Estimated progenitor field sizes for three types of early developmental potency. Totipotent cells give rise to all cells of the developing embryo, including trophectodermal (TE) lineages. Pluripotent progenitors are partitioned into early and late by generation of extra-embryonic endoderm (XEndo) in addition to epiblast (Epi). Dots represent single embryos; solid grey line connects estimates from the same embryo. 


\section{Extended Figure Legends}

\section{Extended Data Figure 1: Target site indel likelihoods from in vitro experiments}

519 a. Histograms for the relative indel frequency for protospacer sites 1,2 , and $2 \mathrm{~b}$ within the target region. In this experiment, single guide RNA expressing vectors respective to each position were delivered into K562 cells. Repair outcomes and frequencies are different for each site, but every site produces hundreds of discrete outcomes. The top 20 most frequent indels for each site are shown. The indel code along the x-axis is as follows: “Alignment Coordinate: Indel Size Indel type (Insertion or Deletion).” Site 3 was not profiled in this experiment.

b. Histograms representing the likelihood that any specific base in the target site is deleted (blue) or has an insertion (red) which begins at that position, for sites 1 and 2, respectively. The position of the integration barcode (intBC) and protospacer sequences (sites) within the target site is represented as a schematic along the bottom, with the PAM

c. Simultaneous and continuous molecular recording of multiple clonal populations in K562 cells. We transduced K563 cells with a high complexity library of unique intBCs, sorted for each site proximal to the intBC. Indels, specifically insertions, start at the double strand break point 3-bases upstream of the PAM sequence. them into wells of 10 cells each and propagated them for 18 days. At the end of the experiment, we detected two populations by their intBCs, implying that only two clonal lineages expanded from the initial population of 10 , and confirmed generation of target site mutations. (Left) Strategy for partitioning a multi-clonal population into their clonal populations. Target sites are amplified from a single cell barcoded cDNA library and the 
intBCs in each cell is identified as present or absent. (Middle) Heatmap of the percent overlap of intBCs between all cells. The cells segregate into two populations representing the descendants of two progenitor cells from the beginning of the experiment. (Right) Table summarizing results of the experiment, including the generation of indels over the experiment duration. These data additionally showcase our ability to combine dynamic recording with tracing based on traditional static barcodes.

Extended Data Figure 2: Capturing early differentiation by pooled sequencing of indels generated within an E9.5 embryo

548 Scatterplots of indel proportions from dissected, bulk tissue of an E9.5 embryo. Placenta is the

549 most distantly related from embryonic tissues, followed by the yolk sac, with the three 550 embryonic compartments sharing the highest similarity.

\section{Extended Data Figure 3: Experimental overview}

a. Schematic of platform used for generation of single cell RNA-seq libraries and corresponding target site amplicon libraries, adapted from Adamson et al., 2016 (Ref 18). The barcoded and amplified cDNA library is split into two fractions prior to shearing: one fraction is used to generate a global transcription profile and the other is used to specifically amplify the target site. 


\section{Extended Data Figure 4: Target site capture in mouse embryos}

564 a. Percentage of cells with at least one target site captured.

565 b. Scatterplot showing the relationship between the mean number of unique molecular identifiers (UMIs, a proxy for expression level) sequenced per target site and the percentage of cells in which the target site is detected, which we refer to as "target site capture." Generally, as the mean number of UMIs increases, the percentage of cells also increases. Using a full length, intron-containing Efla promoter in mouse embryos leads to a higher number of UMIs, which generally results in better target site capture.

c. Percent of cells for which a given integration barcode (intBC) is detected across all seven embryos profiled in this study.

d. Target site capture and expression level across tissues for Embryo 5, which utilizes a truncated Efla promoter to direct transcription of the target site. Each row corresponds to a different intBC, indicated in the top left of the histogram. (Left) The percentage of cells in each tissue for which the target site is captured. (Right) Violin plots represented the distribution of UMIs for the target site in each tissue. Dashed line refers to a $10 \mathrm{UMI}$ threshold. The target site may be expressed at different levels in a tissue-specific manner, sequences carrying the intBCs AGGACAAA and ATTGCTTG may also be explained by mosaic integration after the first cell cycle, as these follow a developmental logic and are preferentially expressed in extraembryonic tissues. White dot indicates the median UMI count for cells from a given germ layer, edges the interquartile range, and whiskers the full range of the data. 
e. Target site capture and expression level across tissues for embryo 7, which drives the target site expression from a full length Ef1a promoter. Each row corresponds to a different intBC, indicated in the top left of the histogram. (Left) The percentage of cells in each tissue for which the target site is captured. (Right) Violin plots represented the distribution of UMIs for the target site in each tissue as in $\mathbf{d}$. Dashed line is a visual threshold for 10 UMIs. While tissue specific expression may explain some discrepancy in target site capture, high expression (as estimated from number of UMIs) may still correspond to low capture rates, as observed for the intBC TGGCGGGG. One possibility is that certain indels may destabilize the transcript and lead to either poor expression or capture.

f. Scatterplots showing the relationship between estimated relative indel frequency and the median number of cells that carry the indel. Since the indel frequency within a mouse is dependent on the timing of the mutation, we cannot calculate the underlying indel frequency distribution using the fraction of cells within embryos that carry a given indel. Instead, we estimate this frequency by the presence or absence of an indel using all of the target site integrations across mice, which reduces biases from cellular expansion but still assumes that any given indel occurs only once in the history of each intBC. Since the number of integrations is small $(<50)$, we might expect our estimates to be poor. Here we see that the number of cells marked with an indel increases with indel frequency, suggesting that our frequency estimates are under-estimated for particularly frequent indels. This is likely due to the fact that we cannot distinguish between identical indels in the same target site that may have resulted from multiple repair outcomes (convergent indels). The most frequent insertions are of a single base and tend to be highly biased 
towards a single nucleotide (eg. 92:1I is uniformly an "A" in 5 out of 7 embryos and never $<88 \%$ ).

611 Extended Data Figure 5: single cell RNA-seq tissue assignment and wild type comparison

612 a. Boxplots representing tissue proportions from E8.0 (top) and E8.5 (bottom) wild type

613 embryos $(\mathrm{n}=10$ each) with lineage-traced embryos mapping to each state overlaid as

614 dots. Wild type embryos display large variance in the proportions of certain tissues and

615 our lineage-traced embryos generally fall within the range of those recovered from wild

616 type. Large circles indicate embryos that were scored as either E8.0 or E8.5,

617 respectively, and the bold red overlay highlights embryo 2, which is used throughout the

618 text. Note that many processes are continuous or ongoing between E8.0 to E8.5, such as

619 somitogenesis and neural development. For example, from E8.0 to E8.5, the embryonic

620 proportions of anterior neural ectoderm and fore/midbrain are inversely correlated as one

621 cell type presumably matures into the other. Many of our embryos scored as E8.0 exhibit

622 intermediate proportions for both tissue types, supporting the possibility that these

623 embryos are somewhat less developed than E8.5 but more developed than E8.0. For

624 boxplots, center line indicates the median, edges the interquartile range, whiskers the

625 Tukey Fences, and crosses the outliers.

626 b. Plots (t-sne) of single cell RNA-seq with corresponding tissue annotations for the six

627 lineage traced embryos used in this study. (Inset) Pie chart of the relative proportions for

628 different germ layers. Mesoderm is further separated to include blood (red). While 36

629 different states are observed during this developmental interval, only broad classifications

630 of certain groups (eg. "neural ectoderm" or "lateral plate mesoderm") are overlaid to 
provide a frame of reference. In general, the relative spacing and coherence of different cell states are consistent across different embryos.

c. Boxplots of the Euclidean distance between single cell transcriptomes and the average transcriptional profile of their assigned cluster (cluster center) in comparison to their distance from the average of the next closest possible assignment. Comparison is to the same 712 informative marker genes used to assign cells to states and includes all cells used in this study. Middle bar highlights the median, edges the interquartile range, whiskers the Tukey Fences, and grey dots the outliers. N's refer to the cumulative number of cells assigned to each state across all 7 embryos for which single cell data was collected, including for embryo 4.

\section{Extended Data Figure 6. Continuous indel generation by breeding}

a. Strategy for generating lineage traced mice through breeding. The target site and guide array cassette are integrated into mouse zygotes as in Figure 2a using C57Bl/6J sperm to generate $\mathrm{P}_{0}$ breeder mice, which are capable of transmitting high copy genomic integrations of the technology. Then, $\mathrm{P}_{0}$ animals are crossed with homozygous, constitutively expressing Cas9 transgenic animals to enable continuous cutting from fertilization onwards in $\mathrm{F}_{1}$ progeny. Shown is Sibling 2 of a cross between a $\mathrm{P}_{0}$ male and a Cas9:EGFP female.

b. Bar charts showing the degree of mutation (\% cut, red) for a $\mathrm{P}_{0}$ male (top row) and $4 \mathrm{~F}_{1}$ offspring generated by breeding with a Cas9:EGFP female prior to weaning (21 days post partum). Each row represents a mouse and each column represents a target site. Each 
sibling inherits its own subset of the 23 parental target site integrations, and demonstrates different levels of mutation throughout gestation and maturation.

c. Indel frequencies for the 10 most frequent indels from 3 siblings in a common target site

\section{Extended Data Figure 7: Performance of tree building algorithms used on embryonic data}

a. Table summarizing contemporary Cas9-based lineage tracers that have been applied to vertebrate development highlighting attributes that differ between the studies. Refer to Methods for a more detailed overview of key characteristics of our technology. * Study reports the average fraction recovered by tissue for integrations that cannot be distinguished, such that percentages reported here are effectively equivalent to our " $\geq 1$ intBC" metric. ** Reports a plate-based DNA-sequencing approach that can be applied to all methods to improve target site recovery. ${ }^{* * *}$ Range of cells where at least one intBC is confidently detected and scored. **** Presents a tree reconstruction method, but results predominantly on clonal analysis.

b. Table of allele complexity, number of nodes, and log-likelihood scores for embryos. Tree likelihoods are calculated using indel frequencies estimated from all embryo data (see Extended Data Figure 5 and Methods). Bold scores indicate the reconstruction algorithm selected for each embryo (see Figure 4, and Extended Data Figures 8 and 9). c. Log likelihood of trees generated using either the greedy or biased sampling approach as a function of complexity, which is measured as the number of unique alleles. There is 
near equivalent performance of the two algorithms for low complexity embryos, but the greedy algorithm produces higher likelihood trees for embryos with larger numbers of unique alleles.

\section{Extended Data Figure 8: Single cell lineage reconstruction of early mouse development for}

\section{embryo 6}

a. Reconstructed lineage tree comprised of 2,690 nodes generated from our most information-dense embryo (embryo 6), which we used to compare shared progenitor scores with embryo 2 in Figure 4d. Each branch represents an independent indel generation event, and each node contains a pie chart of the germ layer proportions for the cells contained within it (colors are as in Figure 3b).

b. Example paths from root to leaf from the selected tree (highlighted by color). Cells for each node in the path are overlaid onto the t-sne representation in Extended Data Figure 5, with the tissue proportion at each node in the tree included as a pie chart. In the top most path (pink), the lineage bifurcates into two independently fated progenitors that either generate mesoderm (secondary heart field/splanchnic plate mesoderm and primitive heart tube) or neural ectoderm (anterior neural ectoderm and neural crest). Note that the middle path (green) also represents an earlier bifurcation from the same tree and eventually produces neural ectoderm (neural crest and future spinal cord). These paths begin with a pluripotent node that can generate visceral endoderm but subsequently lose this potential. Alternatively, the bottom path (dark blue) begins in an equivalently pluripotent state but becomes restricted towards the extraembryonic visceral endoderm fate. 
c. Violin plots representing the relationship between lineage and expression for individual pairs of cells as calculated for embryo 2 in Figure 4c. Expression Pearson correlation decreases with increasing lineage distance, showing that closely related cells are more likely to share function. Red dot highlights the median, edges the interquartile range, and greatest number of unique alleles and samples multiple extraembryonic tissues. Shared whiskers the full range.

d. Comprehensive clustering of shared progenitor scores for Embryo 6, which has the progenitor score is calculated as the sum of shared nodes between cells from two tissues normalized by the number of additional tissues that are also produced (a shared progenitor score is calculated as $2^{-(\mathrm{n}-1)}$ where $\mathrm{n}$ is the number of clusters present within that node). In general, extraembryonic tissues that are specified before implantation, such as extraembryonic endoderm or ectoderm, co-cluster away from embryonic tissues and within their own groups, while the amnion and allantois of the extraembryonic mesoderm cluster with other mesodermal products of the posterior primitive streak. The co-

\section{Extended Data Figure 9: Summary of results from additional mouse embryos}

719 Representative highest likelihood tree analyses for additional embryos, including: 
721 b. Shared progenitor score heatmaps as shown in Figure 5a, normalized to the highest score 722 for each embryo to account for differences in total node numbers. Here, the shared 723 progenitor score is calculated as the number of nodes that are shared between tissues 724 scaled by the number of number of tissues within each node (a shared score is calculated 725 as $2^{-(\mathrm{n}-1)}$ where $\mathrm{n}$ is the number of clusters present within that node). In general, clustering of shared progenitors is recapitulated across embryos, with mesoderm and ectoderm sharing the highest relationship and either extra-embryonic ectoderm or extraembryonic endoderm representing the most deeply rooted and distinct outgroup, though these scores are sensitive to the number of target sites and the rate of cutting. By shared comparison to more represented germ layers. The number of heterogeneous nodes from which scores are derived is included for each heatmap.

734 c. Violin plots representing the pairwise relationship between lineage distance and reflects the rarity of these cells, which restricts them to only a few branches of the tree in transcriptional profile as shown for embryo 2 in Figure 4c. Lineage distance is calculated using a modified Hamming distance and transcriptional similarity by Pearson correlation. The exact dynamic range for lineage distance depends on the number of intBCs included and the cutting rate of the three guide array. Here, distances are binned into perfect ( 0$)$, close $(0>x>0.5)$, intermediate $(0.5 \leq x<1)$, and distant $(x \geq 1)$ relationships for all cells containing either 3 or 6 cut sites, depending on the embryo. As lineage distance increases, transcriptional similarity decreases, consistent with functional restriction over development. Red dot highlights the median, edges the interquartile range, and whiskers the full range. 


\section{Extended Data Figure 10: Expression characteristics of extra-embryonic and embryonic}

\section{6 endoderm}

747 a. Violin plots representing the pairwise scRNA-seq Pearson correlation coefficients for

748 within or across group comparisons according to lineage (X, extra-embryonic; E,

749 embryonic) and cluster assignment (light blue, gut endoderm; dark blue, visceral

750 endoderm). Within group comparisons for cells with the same lineage and transcriptional

751 cluster identity are shown on the left, while across group comparisons are presented on

752 the right. Notably, extraembryonic cells with gut endoderm identities show higher

753 pairwise correlations to embryonic cells with gut endoderm identities (column 4) than

754 they do to visceral endoderm cells, with which they share a closer lineage relationship

755 (column 5). Red dot highlights the median, edges the interquartile range, and whiskers

756 the full range.

757 b. Plots (t-sne) of scRNA-seq data for embryo 2, with gut endoderm cells highlighted.

758 Endoderm cells segregate from the rest of the embryo, and cannot be distinguished by

759 embryonic (light blue) or extraembryonic (dark blue) origin.

760 c. Expression boxplots for the extra-embryonic markers Trap1a and Rhox5 from an

761 independent single cell RNA-seq survey of E8.25 embryos (Ibarra-Soria et al., 2018, Ref

7629 9). Both genes are heterogeneously present in cells identified as mid/hindgut but

763 uniformly present in canonical extra-embryonic tissues, consistent with a subpopulation

764 of cells of extra-embryonic origin residing within this otherwise embryonic cluster. Red

765 lines highlights the median, edges the interquartile range, and whiskers the Tukey Fence.

$766 \quad$ Outliers were removed for clarity. 


\section{Methods}

\section{Plasmid design and construction}

771 Because the principles governing Cas9 efficiency and subsequent indel generation are not

772 absolute, we screened fourteen protospacers for potential inclusion in our target site, including

773 nine protospacers known to function with moderate efficiency and five additional protospacers

774 hypothesized to function ${ }^{43-52}$. Each protospacer was checked against the human and mouse

775 genomes using bowtie to limit off target effects. A gene block library of the fourteen

776 protospacers (no additional bases between sequences) with an 8 base pair randomer was ordered

777 from IDT representing target site version 0.0.

779 The target site (tS) v0.0 vector backbone was derived from a previously described Perturb-seq 780 lentiviral vector (pBA439, Addgene, Cat $\# 85967)^{18}$ with the following changes: the cassette for 781 mU6-sgRNA-EF1a-PURO-BFP was removed and replaced with EF1a-tSv0.0-sfGFP using

782 Gibson assembly with the target site in the coding sequence of sfGFP for use in the fluorescent 783 reporter assay (PCT10, sequence available upon request).

785 A gene block library of five protospacers (ade2-whiteL-bam3-bri1-whiteB; no additional bases

786 between sequences) with an 8 base pair randomer was ordered from IDT representing target site

787 version 0.1. Protospacers in positions 1 (ade2), 3 (bam3), and 5 (whiteB), are used for cutting in 788 subsequent experiments and are referred to as sites 1,2 , and 3.

790 Target site (tS) v0.1 was also cloned into pBA439 with the following changes: the cassette for 791 mU6-sgRNA-EF1a-PURO-BFP was removed and replaced with EF1a-sfGFP-tSv0.1, followed 
792 by BGH pA on the original backbone (PCT12). Here the target site sits in the 3' UTR of GFP.

793 To improve the delivery of multiple targets into the same cell, we swapped the v0.1 target site

794 cassette into a commercially available piggyBac transposon vector (Systems Biosciences,

795 \#PB533A-2) with the following changes: IRES-Neo was swapped for either GFP (PCT16) or

796 mCherry (PCT29). The backbone was digested with restriction enzymes and target site v0.1

797 gene block was PCR-amplified to add Gibson arms. Following Gibson assembly, the plasmids

798 were transformed into at least 100uL of Stbl2 competent cells (Thermo Fisher, Cat\#10268019),

799 and plated onto 1-2 large plates (Fisher, \#NC9372402) with LB/Carbenicillin to generate high

800 complexity target site libraries (PCT17, and PCT30, respectively).

802 The three-guide expression vector design and cloning protocol were adapted from ${ }^{18}$ to utilize

803 guides against the three sites in the target site. The guide for site 1 (ade2) is under the control of

804 the mU6 promoter, site 2 (bam3) under the control of hU6 promoter, and site 3 (whiteB) under

805 the control of bU6-2 promoter. All guides are constitutively expressed in this system.

806 Additionally, the triple-guide cassette was moved onto the piggyBac backbone described above.

807

808 Two further modifications of the plasmids described above were used in this study. First, in an

809 attempt to decrease the cutting percentage variation between embryos, we cloned the triple-guide

810 expression cassette without BFP into PCT29, and then cloned in the target site with intBCs to

811 generate the resulting vectors (PCT41-43, for guide combinations (P,1,P), $(1,1,1)$, and $(2,1,2)$,

812 respectively). In the second modification, we changed the truncated form of Ef1a in PCT29 to a

813 promoter sequence comprised of the ubiquitous chromatic opening element (UCOE) and a full-

814 length, intron-containing Ef1 $\alpha$ and cloned in a triple-guide expression cassette for the guide 
815 combination $(2,1, \mathrm{P})$, followed by cloning in of the target site to make PCT60. In these

816 modifications, target site plasmid libraries (PCT41-43, PCT60) were transformed and expanded

817 in 1-2L of liquid LB/Carb culture rather than on large plates.

818

819 A new target site design, v1.1, was utilized for further experiments to generate $\mathrm{P}_{0}$ breeders (see

820 below). A gene block library of three protospacers (ade2-bri1-whiteB; 30-60 bases between

821 sequences) with a 14 base pair randomer was ordered from IDT representing target site version

822 v1.1. For this target site, site1 is ade2, site2 is bri1, and site3 is whiteB. We cloned v1.1 into the

823 same backbone as PCT60 with guide combinations $(2,3,3)$ or $(2,1,2)$ to make PCT61 and PCT62,

824 respectively.

825

826 Cell culture, DNA transfections, and viral production

827 The production of lentiviral particles or transfection of plasmids as is as described in $^{18}$.

$829 \quad$ K562 GFP reporter assay

830 To construct the target site GFP reporter cell line, a doxycycline(Dox)-inducible Cas9 K562 cell

831 line was stably transduced with PCT10 ( $8 \%$ infected, $<0.1 \mathrm{MOI}$ ), and GFP positive cells were

832 sorted using fluorescence activated cell sorting on a BD FACSAria2. For each protospacer in the

833 target site, 1-4 guides was designed to achieve a series of mutation efficiencies and cloned into

834 single guide expression vectors ${ }^{22}$. On Day -4 , the reporter cell line was plated into wells and

835 stably transduced with a different guide against target site v0.0, GFP-targeting protospacer

836 EGFP-NT2 (positive control), or Gal4-targeting protospacer (negative control) in each well. On

837 Day -2, cells were selected for guide cassette integration using $3 \mathrm{ug} / \mathrm{mL}$ puromycin. On Day 0 , 
$83850 \mathrm{ng} / \mathrm{mL}$ Dox was added to induce Cas9 expression, and maintained through the course of the

839 experiment. GFP fluorescence was recorded on a LSR-II flow cytometer (BD Biosciences) on

840 every $2^{\text {nd }}$ day starting at day 0 , except day 13 was recorded in place of day 12 . Data was

841 analysed in Python using FlowCytometryTools (http://eyurtsev.github.io/FlowCytometryTools/).

842 For guide virus produced in this experiment, labels were systematically shifted during production

843 resulting in incorrect ordering of guide effect on GFP fluorescence, which was corrected for

844 presentation in the manuscript. We confirmed the activity order of the guide series for three

845 guides (ade2, bam3, and bri1) in sequencing experiments where new virus was prepared.

$847 \quad$ K562 single cutting pooled assay

848 To construct the cell line used here, a Dox-inducible Cas9 K562 cell line was stably transduced 849 with PCT12 (6\% infected, $<0.1 \mathrm{MOI})$, and GFP positive cells were sorted on a BD FACSAria2.

850 On Day -5 , the cell line was plated and stably transduced with a different guide against target site 851 v0.1, or GFP-targeting protospacers in each well. On Day -2, cells were selected for guide

852 cassette integration using $3 \mathrm{ug} / \mathrm{mL}$ puromycin. On Day $0,50 \mathrm{ng} / \mathrm{mL}$ Dox was added to induce 853 Cas9 expression, and maintained through the course of the experiment. Wells were sampled 854 every 3-6 days for 20 days with cell pellets frozen down. Genomic DNA was isolated from 855 frozen cell pellets, and the target site was PCR-amplified to make sequencing libraries (refer to 856 Pooled embryo library preparation below for library prep protocol), which were sequenced on 857 the Illumina Miseq. Timepoint samples were pooled and reads with no indels were removed to 858 calculate relative indel frequencies.

$860 \quad$ K562 multiple target site integration cell line 
861 To construct a cell line with multiple integrations, we nucleofected 200,000 Dox-inducible Cas9

862 K562 cells with 1500ng PCT17 and 200ng piggyBAC transposase using set program T-016

863 (Lonza \#V4SC-2096; Systems Biosciences, \#PB210PA-1).

864

865 K562 triple guide cutting assay, and multi-clonal lineage tracing experiment

866 Multiple-integration cells described above were stably transduced with a triple guide expression

867 vector (Perfect-Perfect-Perfect; fastest cutting) and recovered for 2 days. GFP (target site) and

868 BFP (triple guide) double positive cells were sorted using fluorescence activated cell sorting on a

869 BD FACSAria2. For the multi-clonal lineage tracing experiment, 10 cells were sorted into wells

870 containing $200 \mathrm{uL}$ of pre-conditioned media on a 96 well plate (12 wells total). At day 18 , wells

871 were inspected under the microscope and the 3 wells with the largest populations were selected

872 for single cell analysis on the 10x Chromium. Two of the lanes suffered wetting failures, and the

873 remaining sample was taken through library preparation described below (refer to Target site

874 amplicon library preparation). The library was sequenced on the Illumina Miseq and would

875 benefit from additional sequencing.

876

877 For the pooled experiment, $\sim 112,000$ cells were sorted into a tube, spun down, resuspended in

878 fresh media, split into two wells with $50 \mathrm{ng} / \mathrm{mL}$ Dox added to one of the wells. Cells were

879 collected 6 days post-sort, genomic DNA was isolated, and the target site was PCR-amplified to

880 make sequencing libraries (refer to Pooled embryo library preparation), which were

881 sequenced on the Illumina Miseq. The 10 intBCs with the most reads were used for analysis.

$883 \quad$ Embryo and $\mathbf{P}_{\mathbf{0}}$ breeder generation 
884 Protocols are adapted from those described in ref ${ }^{53}$ To enable in vivo lineage tracing, B6D2F1

885 strain female mice (age 6 to 8 weeks, Jackson Labs) were superovulated by sequential

886 intraperitoneal injection of Pregnant Mare Serum Gonadotropin (5IU per mouse, Prospec Protein

887 Specialists) and Human Chorionic Gonadotropin (5IU, Millipore) 46 hours apart. Twelve hours

888 after delivery of the second hormone, MII stage oocytes were isolated and injected with in vitro

889 transcribed piggyBAC transposase mRNA (100 ng/ul) prepared in an injection buffer (5 mM

890 Tris buffer, $0.1 \mathrm{mM}$ EDTA, $\mathrm{pH}=7.4$ ). Decapitated sperm isolated from an 8 week old

$891 G t(R O S A) 26 S o r t m 1.1\left(C A G=\right.$ cas $\left.^{*}, E G F P\right) F e z h / J$ strain mouse (Jackson labs, ref $\left.{ }^{54}\right)$ was

892 resuspended with the purified piggyBAC library in the same injection buffer at concentrations

893 ranging from 0.5 to $1.4 \mathrm{ug} / \mathrm{uL}$.

894 Transposase-injected oocytes were then fertilized by piezo-actuated intracytoplasmic

895 sperm injection (ICSI) as previously described ref ${ }^{55}$. Injected embryos were cultured in $25 \mathrm{uL}$

896 EmbryoMax ${ }^{\circledR}$ KSOM drops (Millipore) covered in mineral oil (Irvine Scientific) in batches of

$89725-50$ embryos. After 84 or 96 hours, successfully cavitated blastocysts were screened for

898 uniform fluorescence of the target sequence cassette and transferred into one uterine horn of 6-10

899 week old pseudopregnant CD-1 strain female mice (Charles River). Uterine transfer results in an

$900 \sim 24$ hour lag, so the day of transfer was scored as E2.5 and embryos were dissected from

901 euthanized animals 6 or 7 days later at $\sim$ E8.5 or E9.5, depending on the experiment. All

902 techniques utilized standard micromanipulation equipment, including a Hamilton Thorne XY

903 Infrared laser, Eppendorf Transferman NK2 and Patchman NP2 micromanipulators, and a Nikon

904 Ti-U inverted microscope.

905 The generation of breeders was conducted identically by coinjecting target design v1.1

906 piggyBAC plasmids with sperm from C57BL6/J strain males (Jackson labs), transferring 
907 uniformly bright mCherry blastocysts into CD-1 strain mice, and allowing live pups to be

908 brought to term. Genotyping was conducted using tail tip genomic DNA purified using the

909 Quick DNA Miniprep Plus kit (Zymogen) isolated prior to weaning. Animals with large intBC

910 counts ( $\mathrm{n}=23$ for the male used in Extended Data Figure 6) were then bred into either male or

911 female $G t(R O S A) 26 \operatorname{Sortm} 1.1\left(C A G=\operatorname{cas} 9^{*}, E G F P\right) F e z h / J$ strain animals to generate live pups

912 with continuous cutting. Fluorescence of live animals was confirmed and documented using a

913 dual fluorescent protein flashlight (Nightsea).

914

\section{$915 \quad$ Pooled embryo library preparation}

916 RNeasy Mini Kit (Qiagen, \#74104) was used to isolate RNA from whole embryos or dissected

917 tissue for embryonic tissue. Alternatively, genomic tail tip DNA was used for $\mathrm{P}_{0}$ breeders or

918 Cas9 $+\mathrm{F}_{1}$ animals. Following purification and/or first strand synthesis of cDNA from 1 ug of

919 RNA (Promega), the target site was amplified using a 2-stage PCR protocol. In the $1^{\text {st }}$ stage,

$920<100$ ng of diluted DNA template was amplified using $0.6 \mathrm{uM}$ forward and reverse primers and

921 Kapa HiFi HotStart ReadyMix according to the following PCR protocol: (1) 98C for 3 min, (2)

$92298 \mathrm{C}$ for $30 \mathrm{~s}, 69 \mathrm{C}$ for $30 \mathrm{~s}, 72 \mathrm{C}$ for $15 \mathrm{~s}$ ( 16 cycles for cDNA, 24 cycles for genomic DNA), (3)

$92372 \mathrm{C}$ for $5 \mathrm{~min}$. Following 0.7X SPRI selection, the elute served as template for $2^{\text {nd }}$ stage PCR,

924 using 0.6uM barcoded P5 and P7 secondary primers and Kapa HiFi HotStart ReadyMix

925 according to the following PCR protocol: (1) 98C for 3 min, (2) $98 \mathrm{C}$ for $30 \mathrm{~s}, 60 \mathrm{C}$ for $30 \mathrm{~s}, 72 \mathrm{C}$

926 for $30 \mathrm{~s}$ (4-6 cycles), (3) $72 \mathrm{C}$ for $5 \mathrm{~min}$. PCR products underwent $0.6 \mathrm{X}$ SPRI-selection and were

927 eluted in $20-40 \mathrm{uL}$ of elution buffer to produce the final library. Libraries were sequenced on the

928 Illumina HiSeq 2500 (Rapid Run) or Miseq, with the following run parameters: Read 1: 175

929 cycles, i7 index: 8 cycles, i5 index: 8 cycles, Read 2: 175 cycles. 
931 For v1.0 target sites, the following primary primers were used:

932 MC38:

933 CGTCGGCAGCGTCAGATGTGTATAAGAGACAGTGCAGGAGCGGATTGCTTCGAACC

934 MC39:

935 TCTCGTGGGCTCGGAGATGTGTATAAGAGACAGACAACCACTACCTGAGCACCCAG

936 TC

937 For v1.1 target sites, the following primary primers were used:

938 P5_PCT48-49_F:

939 TCGTCGGCAGCGTCAGATGTGTATAAGAGACAGAATCCAGCTAGCTGTGCAGC

940 ODY120_PCT48_R_PB:

941 GTCTCGTGGGCTCGGAGATGTGTATAAGAGACAGGCGATGGACGATTGCGGAAGAC

942 AG

943 Secondary amplification was conducted using the following primers:

944 P5 primer:

945 AATGATACGGCGACCACCGAGATCTACAC[ILLUMINA

946 INDEX]TCGTCGGCAGCGTCAGATGTGTA)

$947 \quad$ P7 primer

948 CAAGCAGAAGACGGCATACGAGAT[ILLUMINA

949 INDEX]GTCTCGTGGGCTCGGAGATGTGTATAAG

950

951 Single cell embryo dissociation 
952 Embryos are washed through several drops of PBS after isolation to reduce debris and put into

$953 \sim 100$ uL PBS droplets on a microscope slide and screened for uniform fluorescence of the target

954 site cassette on an Olympus IX71 inverted microscope running Metamorph. Selected embryos

955 were dissociated to single cell suspensions by adding $100 \mathrm{uL}$ of TrypLE (Invitrogen, \#12605010)

956 and pipetting the embryo or embryo pieces every 5 minutes for $\sim 30$ minutes until complete

957 dissociation was visually confirmed. Trypsin was deactivated by adding $100 \mathrm{uL}$ PBS+BSA is

958 added to the droplet and moving cells into a $1.5 \mathrm{~mL}$ eppendorf tube, followed by several rounds

959 of additional collection with 100 to $200 \mathrm{uL}$ of PBS+BSA to a final volume of $1 \mathrm{~mL}$. The

960 dissociated cells are filtered through a Flowmi filter tip (Bel-Art Products, \#H13680-0040) into a

961 new tube, and spun down for 5 minutes at $1200 \mathrm{rpm}$ on a tabletop centrifuge. Following the

962 spin, $900 \mathrm{uL}$ of PBS+BSA is removed and the remaining volume is resuspended with an

963 additional $900 \mathrm{uL}$ of PBS+BSA. The suspension is spun for 5 minutes at $1,200 \mathrm{rpm}, 800 \mathrm{uL}$ of

964 PBS+BSA is removed, the remaining volume is spun for 5 minutes at $1,200 \mathrm{rpm}$, and PBS+BSA

965 is removed until only $\sim 30 \mathrm{uL}$ of volume remains. $2 \mathrm{uL}$ of the final resuspended cells were used

966 for counting using a hemocytometer. We load $\sim 17,000$ cells into the 10x machine (Chromium

967 Single Cell 3' Library \& Gel Bead Kit v2) for a targeted recovery of 10,000 cells.

968

969 scRNA-seq library preparation and sequencing

970 Single cell RNA-seq libraries were prepared according to the 10x user guide, except for the

971 following modification. After cDNA amplification, the cDNA pool is split into two fractions.

$97215 \mathrm{uL}$ of EB buffer is added to one of the fractions of $20 \mathrm{uL}$ of the cDNA pool, and scRNA-seq

973 library construction proceeds as directed in the 10x user guide. RNA-seq libraries were

974 sequenced on the Illumina HiSeq 4000 system. 


\section{Target site amplicon library preparation}

977 The target site-specific amplification protocol was adapted from ${ }^{11}$. 50-100 ng of template from

978 the cDNA pool, 0.3 uM P5-truseq-long

979 (AATGATACGGCGACCACCGAGATCTACACTCTTTCCCTACACGACGCTCTTCCGATC

980 T), 0.6 uM MC63

981 (TCTCGTGGGCTCGGAGATGTGTATAAGAGACAGTGCAGGAGCGGATTGCTTCGAAC

982 C) was split across four parallel PCR reactions, and was amplified using Kapa HiFi HotStart

983 ReadyMix according to the following PCR protocol: (1) 95C for $3 \mathrm{~min}$, (2) 98C for $15 \mathrm{~s}$, then

984 69C for $15 \mathrm{~s}$ (8-12 cycles). Reactions were re-pooled during 0.9X SPRI selection, and eluted

985 into $60 \mathrm{uL}$. A second PCR with the elute as the template, $0.3 \mathrm{uM} \mathrm{P5}$

986 (AATGATACGGCGACCACCGA), 0.6 uM barcoded P7

987 (CAAGCAGAAGACGGCATACGAGAT[ILLUMINA

988 INDEX]GTCTCGTGGGCTCGGAGATGTGTATAAG) was split across four parallel PCR

989 reactions, and amplified using Kapa HiFi HotStart ReadyMix according to the following PCR

990 protocol: (1) 95C for $3 \mathrm{~min}$, (2) 98C for $15 \mathrm{~s}$, then 69C for $15 \mathrm{~s} \mathrm{(6} \mathrm{cycles).} \mathrm{Reactions} \mathrm{were} \mathrm{re-}$

991 pooled during 0.9X SPRI selection and then fragments of length 200-600bp were selected using

992 the BluePippin. Target site libraries were sequenced on the Illumina HiSeq 2500 (Rapid Run),

993 with the following run parameters: Read 1: 26 cycles, i7 index: 8 cycles, i5 index: 0 cycles, Read

994 2: 350 cycles.

995

996 scRNA-seq library data processing 
997 scRNA-seq data was processed and aligned using 10x Cell Ranger v2. The filtered gene-barcode

998 matrices were then processed in Seurat (https://satijalab.org/seurat/) for data normalization

999 (global scaling method "LogNormalize"), dimensionality reduction (PCA), and generation of t-

1000 sne plots, which use the first 16 principal components.

1001

1002 scRNA-seq tissue assignment

1003 An independent project conducting scRNA-seq profiling of gastrulation identified 42 distinct

1004 tissues in wild type mice. We utilized the mean expression profile for each tissue and the list of

1005712 marker genes used for assignment of cells to tissues (see instructions for assignment in

1006 GSE122187). For each cell in lineage traced mouse embryos, we calculated the Euclidean

1007 distance between the cell's expression profile and the mean expression profile for each tissue

1008 using the 712 marker gene set, and assigned the cell to the tissue identity with the minimum

1009 distance. Expression values were transformed to log space using $\log ($ normalized UMI count +1$)$

1010 before calculating the Euclidean distance. Comparisons between the best matched tissue to the

1011 next best match are presented for all data collected here in Extended Data Figure 5 to highlight

1012 the precision of this approach.

1013

\section{Embryo gastrulation stage assignment}

1015 The wild type mouse gastrulation compendium consists of five time points, profiling every 0.5

1016 days from E6.5 to E8.5 with at least 10 embryos collected for each time point. Tissue proportion

1017 is calculated as the number of cells assigned to the tissue divided by the total number of cells in

1018 the embryo. The median tissue proportion was calculated for each time point treating each tissue

1019 independently. For each lineage-traced embryo, the Euclidean distance between its tissue 
1020 proportions and the median tissue proportion for each time point was calculated and the embryo

1021 was assigned to the time point with the minimum cumulative distance. All lineage-traced

1022 embryos were assigned to either E8.0 or E8.5 stages.

1023

1024 Target site data processing

1025 A custom software pipeline was built to align and call indels in the target site. The logic is as

1026 follows: (1) Assign cell barcode and UMIs to each read, (2) find the consensus sequence for each

1027 UMI, (3) align the consensus sequence to the target site reference sequence, (4) identify most

1028 likely integration barcodes (intBC) and create custom reference sequences, (5) repeat alignment

1029 against all reference sequences and select highest scoring alignment for each UMI, (6) call intBC

1030 and indels in the target site, (7) correct the intBC and allele using UMIs which appear in the

1031 same cell, (8) remove doublets. Details appear below:

1032

1033 (1) Assigning cell barcode and UMIs to each read. Specific amplification libraries of the target

1034 site amplicon were processed using 10x Cell Ranger software to assign cell barcodes and UMIs

1035 to each read. The target site is designed to be orthogonal to the human and mouse genome, and

1036 does not align in Cell Ranger processing. Unaligned reads from the Cell Ranger output bam file

1037 are parsed into fastq format with the cell barcode and UMI identifiers appended to the read 1038 name.

1039

1040 (2) Finding the consensus sequence for each UMI. To potentially increase the speed of consensus

1041 sequence finding, we attempt to trim reads to the same length for each UMI. The read is

1042 trimmed to remove sequence beyond the polyA tail using cutadapt software 
1043 (http://cutadapt.readthedocs.io/en/stable/) with the following parameters: [-a AAAAAAAAAA -

1044 e $0.1-$ o trimmedFile.fq - untrimmed-output $=$ untrimmedFile.fq - m $20-$ max-n=0.3 - trim-n].

1045 Reads that do not contain polyA sequence appear in the untrimmed file and are subjected to a

1046 second round of read trimming using a sequence which appears in the 3' end of the target site

1047 assuming the sequence has not been deleted from DNA repair, with cutadapt run using the

1048 following parameters: [-a GCTTCGTACGCGAAACTAGCGT -e 0.1 -o trimmedFile2.fq --

1049 untrimmed-output=untrimmedFile2.fq -m 20 --max-n=0.3 --trim-n --no-indels]. The adapter

1050 sequence used in the last round of trimming is then concatenated back on to the trimmed

1051 sequence to improve target site alignment in the next step. If $>=60 \%$ of trimmed sequences for a

1052 given UMI are the same sequence, then the sequence is taken as the consensus sequence.

1053 Otherwise, a multiple sequence alignment is performed using BioPython and the consensus

1054 sequence is extracted from the alignment. Ambiguous bases are reported if there is $<50 \%$

1055 agreement for any position in the alignment.

1056

1057 (3) Aligning to the target site reference. We use the emboss implementation of the smith-

1058 waterman algorithm to align sequences to the target site reference sequence with the following

1059 parameters, which were determined empirically: [emboss water -asequence targetSiteRef.fa -

1060 sformat1 fasta -bsequence consensusUMI.fa -sformat2 fasta -gapopen 15.0 -gapextend 0.05 -

1061 outfile sam -aformat sam]. In this first alignment, the ambiguous sequence NNNNNNNN is

1062 used to represent the intBC. A minor bug had to be corrected in the emboss implementation to

1063 successfully output sam format. For target site v1.1, the gapopen penalty was increased to 20

1064 and the gapextend penalty to 1 .

1065 
1066 (4) Identifying the most likely intBC. A perl script is used to parse the intBC from the alignment.

1067 The intBCs with the highest number of UMIs are substituted into the target site reference

1068 sequence to make custom reference sequences. This step was included because upon manual

1069 inspection, there were obvious misalignments due to the ambiguous intBC sequence, which were

1070 corrected upon substitution of a real sequence.

1071

1072 (5) Selecting the highest scoring alignment for each UMI. Repeat smith waterman alignment

1073 against all custom reference sequences and select alignment with the highest score for each UMI.

1074

1075 (6) Calling indels and intBCs. A perl script is used to parse the intBC and indels from the

1076 alignment using the CIGAR string. The boundaries for each site is defined and indels

1077 overlapping site boundaries are called as an indel in that site. Sequence of the indel is not

1078 considered.

1079

1080 (7) Correcting indels using multiple reads with the same UMI from the same cell. UMIs are

1081 filtered for alignment score and only cells that are in the matched scRNA-seq data set are kept.

1082 An intBC is corrected to an intBC with a higher UMI count in the cell if the intBCs are within an

1083 edit distance of 2 and the alleles are the same. An allele is the combination of indels in sites 1,2,

1084 and 3. An allele is corrected to an allele with a higher UMI count in the cell if the intBC is the

1085 same and the allele is within a 1-indel difference. Only UMIs with greater than or equal to 3

1086 UMIs are kept.

1087 
1088 (8) Eliminating doublets. Cells that report two alleles for the same intBC are removed if the 1089 dominant allele is $<80 \%$ of the total UMI count for the intBC. This removes $4.1-18.3 \%$ of cells 1090 in our embryos.

1091

1092 Tree reconstruction strategies

1093 1. Biased search through phylogenetic space

1094 We simulate the evolutionary process leading from a collection of uncut target sites to the final

1095 data set. The set of mutations (including "no mutation") across all target sites in a cell is referred

1096 to as an allele. In the final tree, each branch represents a mutation, and each node represents the

1097 allele of a cell, which may be a reconstructed ancestral allele, i.e. it is not present in the data set.

1098 Input: table of unique alleles

1099 - each allele may represent multiple cells

1100 - we cannot distinguish between identical indels in the same position that may result from

1101 independent mutation events (convergent indels) if they appear with an identical set of co-

$1102 \quad$ segregating indels

1103 Algorithm:

1104 - Create root node in tree representing an allele with 0 mutations (c_allele)

1105 - remove alleles in the table that match c_allele

1106

1107 - While alleles remain in table:

1108 - choose indel from table that can be added to current allele

$1109 \quad$ - can only add indels in positions that have no mutation

1110 - create new node by adding indel into c_allele (c_allele2) 
1111 - draw directed edge labeled with indel between nodes from c_allele to c_allele2

1112 - remove alleles in table that match c_allele2

1113 - includes alleles that match c_allele2 with missing values for positions that have no

1114 mutations

1115 - if indels in table can be added to c_allele2, then c_allele $=\mathrm{c} \_$allele2; else, $c \_$allele does

1116 not change

1117 - when indels cannot be added to c_allele, traverse up edges to ancestral nodes until an

1118 allele to which an indel can be added is found

1120 We presented two methods that are used to choose indels. The first method, "Random," selects a

1121 position where an indel can be added, and then selects an indel from the data set for that position;

1122 both selections occur in an unbiased manner. The second method, "Frequency Normalized

1123 Weighted" (FNW), identifies all of the indels that can be added to the current allele and scores

1124 them according to the fraction of alleles they are found in divided by the expected independent

1125 frequency of the indel (see Fig. 2c). These scores are used as weights to bias selection of the

1126 indel. The reasoning behind FNW is that indels that are found in many cells (or alleles) are more

1127 likely to have occurred early, but this has to be balanced against their expected likelihood of

1128 occurring. FNW biases the search towards more likely trees. To further increase the search for

1129 good trees, we first remove all indels that are unique to a single allele since we can assume that

1130 these indels occur at the leaves of the tree. The indels are added as branches leading to leaves in

1131 the final tree before the final tree likelihood is calculated.

1132 
1133 The log likelihood of the tree is calculated as the sum of the likelihoods of all the indels that

1134 appear in the tree. The likelihood of each mutation is estimated from the embryo data set (Fig.

1135 2c).

1136

1137 It is worth noting that the number of trees that are possible grossly exceeds 30,000 ; however, the

1138 search is biased towards finding good trees and performs markedly better than those that are

1139 randomly generated. Using high scoring trees to direct the search towards better ones, such as by

1140 grafting high scoring branches, could further improve our algorithm's ability to identify high

1141 scoring trees.

\section{2 . Greedy search to reconstruct larger trees}

1144 Our greedy algorithm consists of building the tree top-down, recursively splitting cells into

1145 mutually exclusive groups based on the presence or absence of a specific mutation. In particular,

1146 these splits are prioritized by selecting mutations that appear frequently in the dataset, but are

1147 known to be an improbable outcome from a Cas9 mutagenesis event. This transform is equal to

1148 the product of the observed frequency of the mutation and the log prior-probability. The

1149 mutations prioritized this way, we reason, are very likely to have occurred only once and

1150 relatively early in the experiment. Under this assumption, these mutations are useful to a top-

1151 down approach as they efficiently create maximally informative tree-splits. In practice, we can

1152 calculate the prior-probabilities of mutations several ways but while describing this algorithm we

1153 assume the priors are provided (Fig. 2c).

1154 
1155 To deal with missing values, we first split cells based on the presence or absence of a mutation.

1156 Then, for each cell that reports a missing value for this cut site, we assign the cell to the group

1157 with which it shares the greatest similarity. Here, we define similarity as the average number of

1158 mutations it shares with the cells in each group. We follow this procedure until only one cell

1159 remains. Note that for application to the dataset described in this manuscript, we filled missing

1160 values with unique indels to force the algorithm to choose splits based on the presence of

1161 mutations rather than absence.

1163 Theoretically, building the tree in this fashion is possible due to the special case of multistate

1164 compatibility afforded by our model of evolution, namely that mutations can only arise once at a

1165 particular site (i.e. Cas9 cannot re-cut a site). This context allows one to consider a traditional

1166 Gusfield algorithm ${ }^{56}$ in which one infers phylogenies by selecting character-splits based on the

1167 most frequently occurring mutations. In a special regime of "perfect-phylogeny" (where every

1168 mutation arose exactly once), this algorithm is provably optimal and extremely efficient as

1169 compared to other algorithms (linear in the number of cells and mutations, or O $(\mid$ number cells $\mid$ *

1170 |number of mutations|)). In the case of multi-state characters, the notion of compatibility often

1171 breaks down as this typically implies that a character can mutate many times to different states.

1172 Yet, as described previously, in our system this cannot happen - namely, once a mutation is

1173 obtained at a site, it cannot be changed again along that evolutionary path. In this way, we can

1174 apply an approximated Gusfield algorithm to reconstruct trees, where perfect phylogeny is

1175 possible although still confounded in cases where the same mutation arises twice independently.

1177 Trees are visualized using the Python ete library (http://etetoolkit.org/). 


\section{Pairwise single cell lineage distance measure used for violin plots}

1180 A cut site can take 2 forms, uncut or indel. The distance is a modification of hamming distance

1181 where uncut is considered a special state.

1182 Distance $=(2 *($ sites with different indels $)+1 *($ sites with indel vs uncut $)) /($ number of sites

1183 recovered in both cells)

1184 Pairwise expression correlation was estimated using the same 712 marker genes used to assign

1185 cell states and was only included if two single cell transcriptomes shared $\geq 10$ gene

1186 measurements.

1188 Estimating ancestral tissue relationships

1190 Each node, including leaves, that includes more than one tissue type is considered a

1191 "progenitor." Progenitors found at the leaves are not reconstructed or inferred but result from the

1192 lack of new indels that distinguish between tissues (ie. the lineage tracer does not produce new

1193 indels past the progenitor stage).

1195 The shared progenitor score is calculated between two tissues as the number of shared

1196 progenitors scaled by the number of tissues each progenitor contributes to, and is calculated

1197 using the following algorithm:

1198

1199 For each progenitor,

$1200 \quad$ tList $=$ list of tissues progenitor contributes to 
pScore $=1 /\left(2^{\wedge} \operatorname{len}(\right.$ tList $\left.)-1\right)$

for each pair of tissues in tList:

$$
\text { progenitorScoreForPairOfTissues }+=\text { pScore }
$$

1204 Example for a single progenitor:

$$
\text { tList }=[\text { Endo, Meso, XMeso }]
$$

$$
\text { pScore }=1 /\left(2^{\wedge}(3-1)\right)=1 / 4
$$$$
\text { ProgenitorScoreEndoMeso }+=1 / 4
$$$$
\text { ProgenitorScoreEndoXMeso }+=1 / 4
$$$$
\text { ProgenitorScoreMesoXMeso }+=1 / 4
$$

1211 The resulting matrix is a shared progenitor score matrix. To transform the similarity matrix to a 1212 distance matrix, we use 1-(matrix/maxScoreInMatrix). The distance matrix is then hierarchically 1213 clustered using either ward or average as the cluster joining criteria..

1215 To account for the potential effect of cluster sizes (for example, if we assume that differentiation

1216 occurs for all tissues instantaneously, then the larger cluster sizes for mesoderm and ectoderm

1217 would increase the likelihood of detecting a progenitor between the two tissues), we

1218 downsampled each tissue before calculating the shared progenitor score: 150 cells were

1219 randomly sampled from each tissue and the tree was pruned to only include the sampled cells.

1220 For tissues with less than 150 cells, all cells were included. For embryo 2, we downsampled to 1221300 cells since it is a merger of two biological replicates and is therefore doubly sampled. The

1222 shared progenitor score was calculated from the pruned tree and the process was repeated 1000 1223 times for each embryo. The median progenitor score is presented in the heatmap. For higher 
1224 resolution clusters (Fig. 4d, Extended Data Fig. 8), we downsampled 500 times instead of 1000

1225 times.

1227 Note that the number of nodes reported below the heatmaps in Extended Data Figure 8

1228 represents the number of progenitors that are found in the complete tree. The number of nodes

1229 used to calculate the shared progenitor score depends on the sampled set of cells chosen.

1230 For high resolution shared progenitor scores calculated for embryos 2 and 6 (Fig. 4d and

1231 Extended Data Fig. 8), we bolstered some populations prior to calculating shared progenitor

1232 scores by merging some cluster identities if they represent the linear maturation of one tissue

1233 type to another, are primarily one cluster versus the other at the assigned developmental time

1234 point, and have very close transcriptional profiles. Specifically, we merged node with

1235 notochord, amnion mesoderm (early) with amnion mesoderm (late), primitive blood progenitor

1236 with primitive blood (early), and anterior paraxial with pharyngeal (arch) mesoderm. We also

1237 merged surface and preplacodal ectoderm due to the similarity of their transcriptional profiles

1238 and omitted "similar to neural crest 2" as this transcriptional cluster is ambiguously determined

1239 (the cluster is globally most similar to neural crest but not obviously comprised of specific

1240 marker genes).

1242 Endoderm lineage assignment and differentially regulated gene identification

1243 Endoderm cells can have one of three origins based upon our tree: extra-embryonic, embryonic,

1244 or ambiguous. Cells are considered extra-embryonic if there is a progenitor in its lineage whose

1245 descendants include $\geq 40 \%$ extra-embryonic cells. Cells have ambiguous origin if they descend 
1246 directly from the root node. Otherwise, cells are considered to be from embryonic origin. We

1247 identified endoderm cells of extra-embryonic origin in all embryos but embryo 7.

1249 We use the Kolmogorov-Smirnov test (Python scipy.stats.ks_2samp) to identify differentially

1250 regulated genes between embryonic and extra-embryonic origin endoderm cells. Only highly

1251 variable genes in the embryo are considered for testing, and genes are significant if they have a

1252 Bonferroni corrected p-value under 0.05.

\section{Multipotent field size estimation and asymmetry}

1255 Progenitors are considered pluripotent if their descendants include at least one mesoderm (Meso

1256 or XMeso or Blood) cell, one ectoderm (Ecto) cell, and one endoderm (Endo) cell. A pluripotent

1257 progenitor are considered early pluripotent if it also has at least one extra-embryonic endoderm

1258 descendant, and further considered totipotent if it has at least one extra-embryonic ectoderm

1259 descendant. To estimate the lower bound for the number of multipotent cells, we start at the

1260 bottom level of the tree and count the number of multipotent cells at that level. If multipotent

1261 cells exist, then the number of multipotent cells is propagated to its ancestor in the above level,

1262 otherwise we count 0 for that level. We add one progenitor for every level that includes a

1263 multipotent cell and other cells to represent the progenitor that lead those non-multipotent cells at 1264 that level. The number of multipotent cells is then the number of cells propagated to the root of 1265 the tree. Progenitor asymmetry is simply the proportion of descendants from each of the tissues 1266 for that node.

1267 Comparison to other technologies 
1268 Several CRISPR-Cas9 based lineage tracers have been developed, each with distinct strengths

1269 and weaknesses. In Extended Data Figure 7, we present a table summarizing the different

1270 technologies, and elaborate on the attributes that, in combination, distinguish our strategy here:

1271 1. Target sites are marked with a unique integration barcode (intBC). The intBC allows us to

1272 phase our target sites and perform a direct comparison for each target site across cells. This

1273 greatly improves the information content of our system as it allows us to distinguish between

1274 the same indel if it appears in different target sites (Fig. 1c).

1275 2. Guide RNAs are integrated into genomic DNA and constitutively expressed from

1276 totipotency, which enables our lineage tracer to be truly evolving over multiple cell

1277 generations. In technologies applied to zebrafish development, guideRNAs are expressed as

1278 a pulse, which leads to the generation of a large diversity of barcodes at one or two

1279 timepoints.

1280 3. Multiple integrations of multi-cutsite target sites are distributed throughout the genome.

1281 Technologies that integrate a single target site with many cut sites or have tandem

1282 integrations are subject to collapse of information when one indel may affect neighboring cut

1283 sites or alternatively, simultaneous cutting of several cut sites remove large portions of their

1284 lineage tracer. While our technology is also vulnerable to these effects, we are better

1285 buffered against them by distributing the target sites throughout the genome. We also

1286 highlight that indel generation is largely independent within target sites when slower cutters

1287 are used (Fig. 2d-f).

1288 4. Simultaneous, multi-population lineage tracing (Extended Data Figure 1c). Since target

1289 sites are labeled with integration barcodes, we can use the identity of these barcodes to

1290 deconvolute pools of cells upon sequencing. Alternatively, independent samples, such as 
1291 embryos that have unique sets of integration barcodes, can be pooled onto a single 10x lane

1292 to decrease the cost of reagents.

1293 5. Multi-channel recording using our triple guide vector. In our current manuscript, we use the

1294 three channels for lineage tracing but different types of sensors can be developed to record

1295 multiple independent inputs.

1296 6. Ability to trace over different time scales by tuning the mutation rate of the system through

1297 mismatches in the guide RNA.

1298 To fully utilize the information captured in our data set, we developed custom reconstruction

1299 strategies to identify the maximum likelihood tree (see Tree reconstruction strategies

1300 above). We estimate indel likelihoods using all of our embryo data (Fig. 2c), which allows us to

1301 estimate tree likelihoods rather than utilize maximum parsimony criteria. Phylogenetic

1302 algorithms developed for tumor evolution, such as $\mathrm{SCITE}^{57}$, offer conceptual frameworks that

1303 are compelling to adapt for our technology.

1304

1305 Code availability

1306 Code will be shared upon request.

1307 Data Availability

1308 The data is available in the GEO database under accession numbers GSE117542 for lineage

1309 traced embryos and GSE122187 for the gastrulation compendium.

1310

1311

1312 


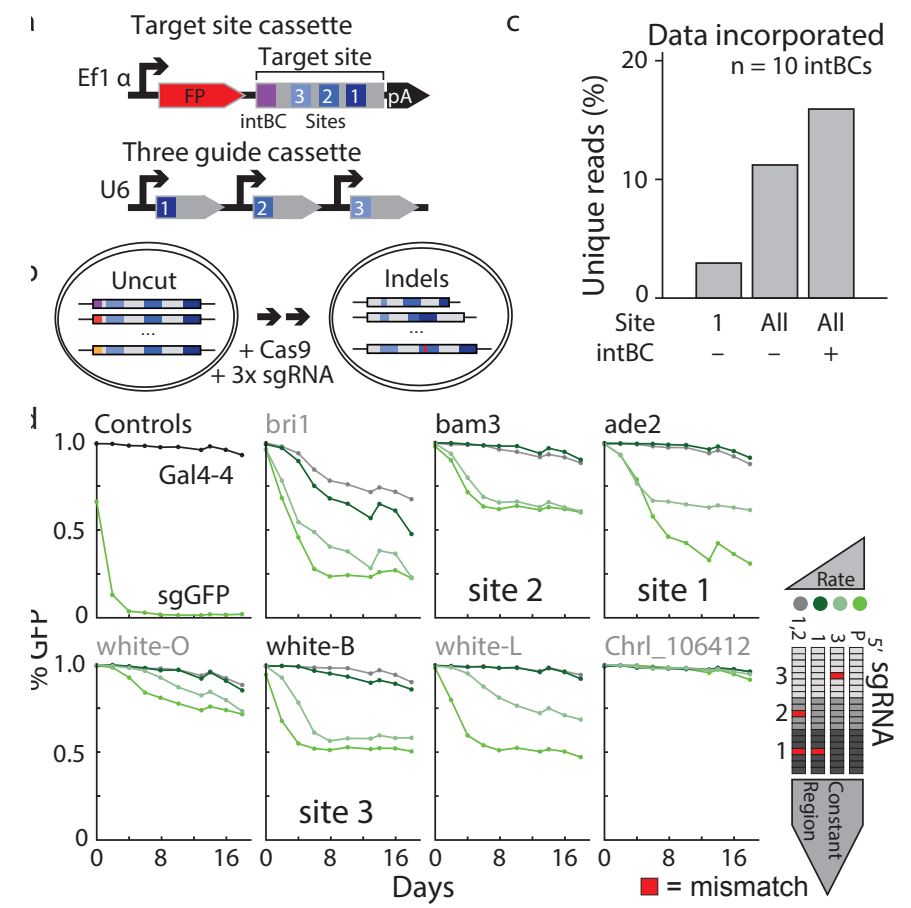



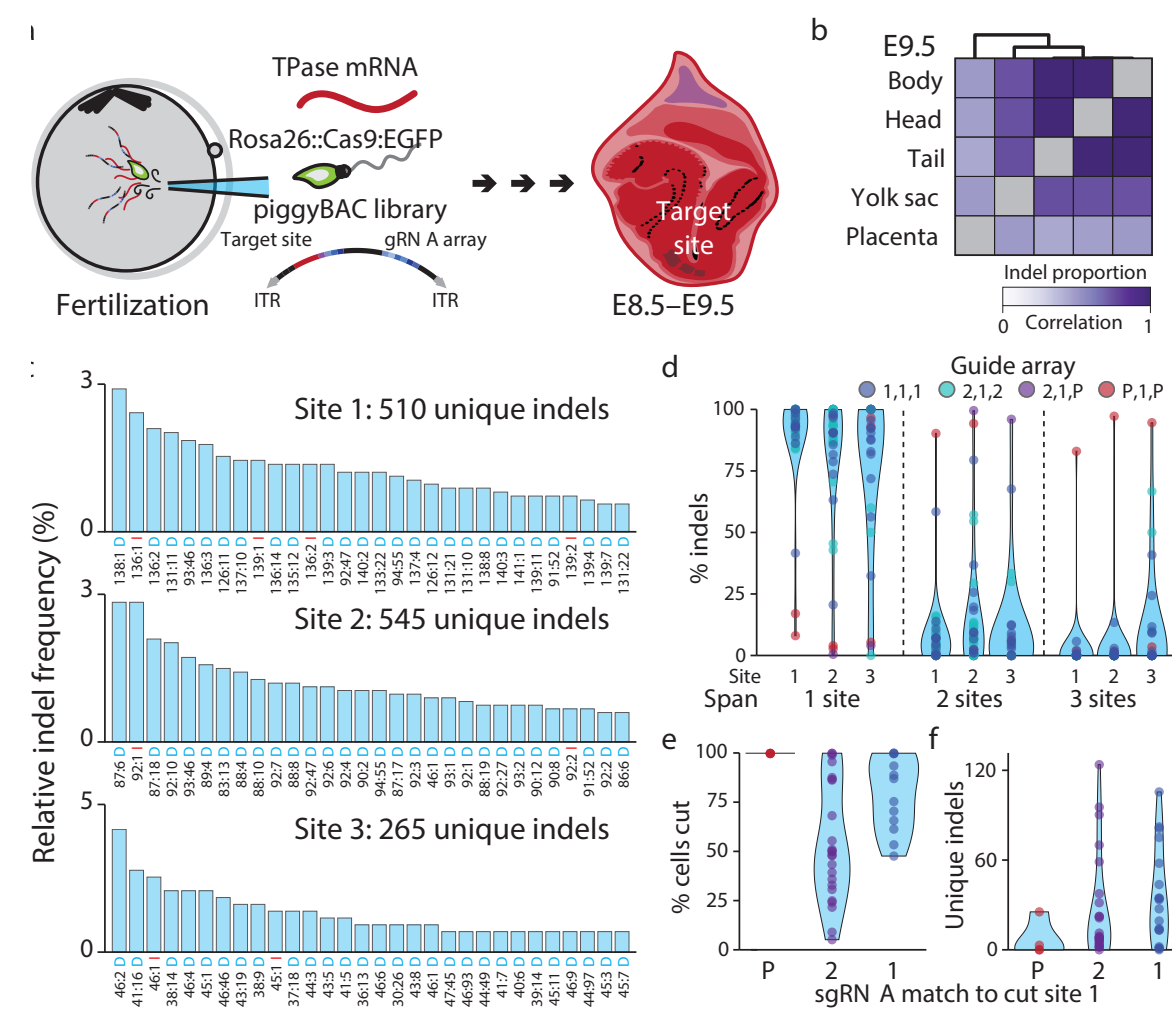

d Guide array
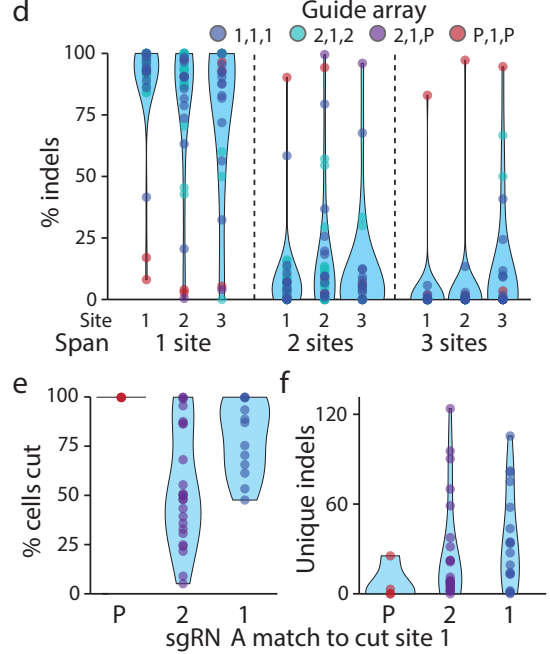


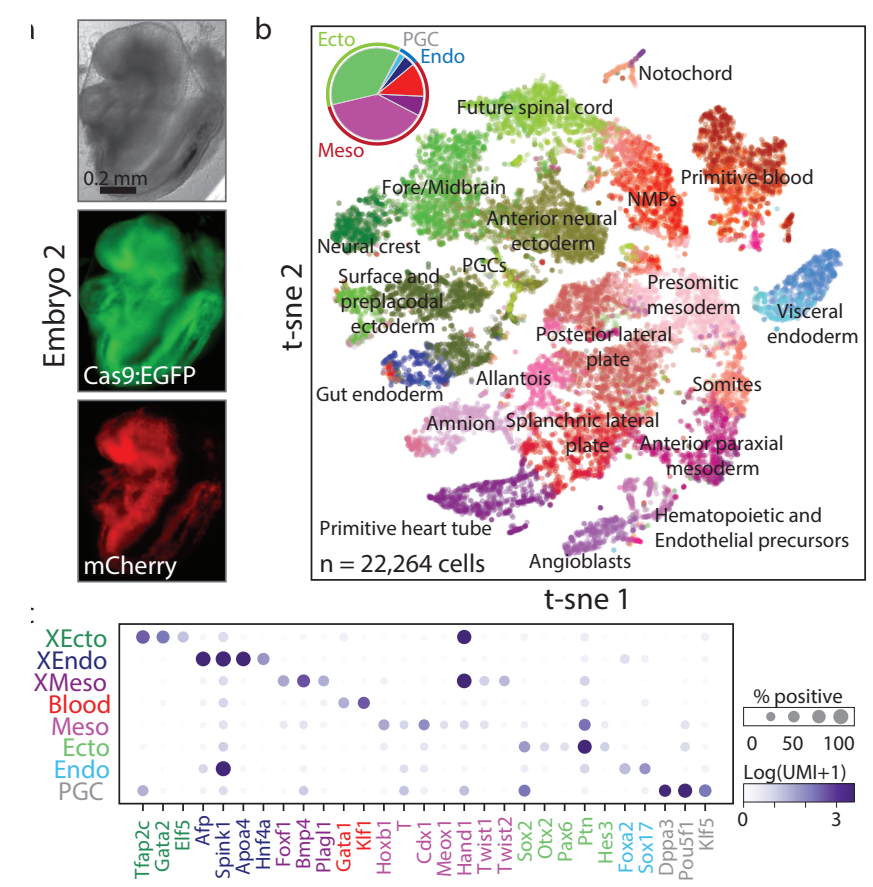




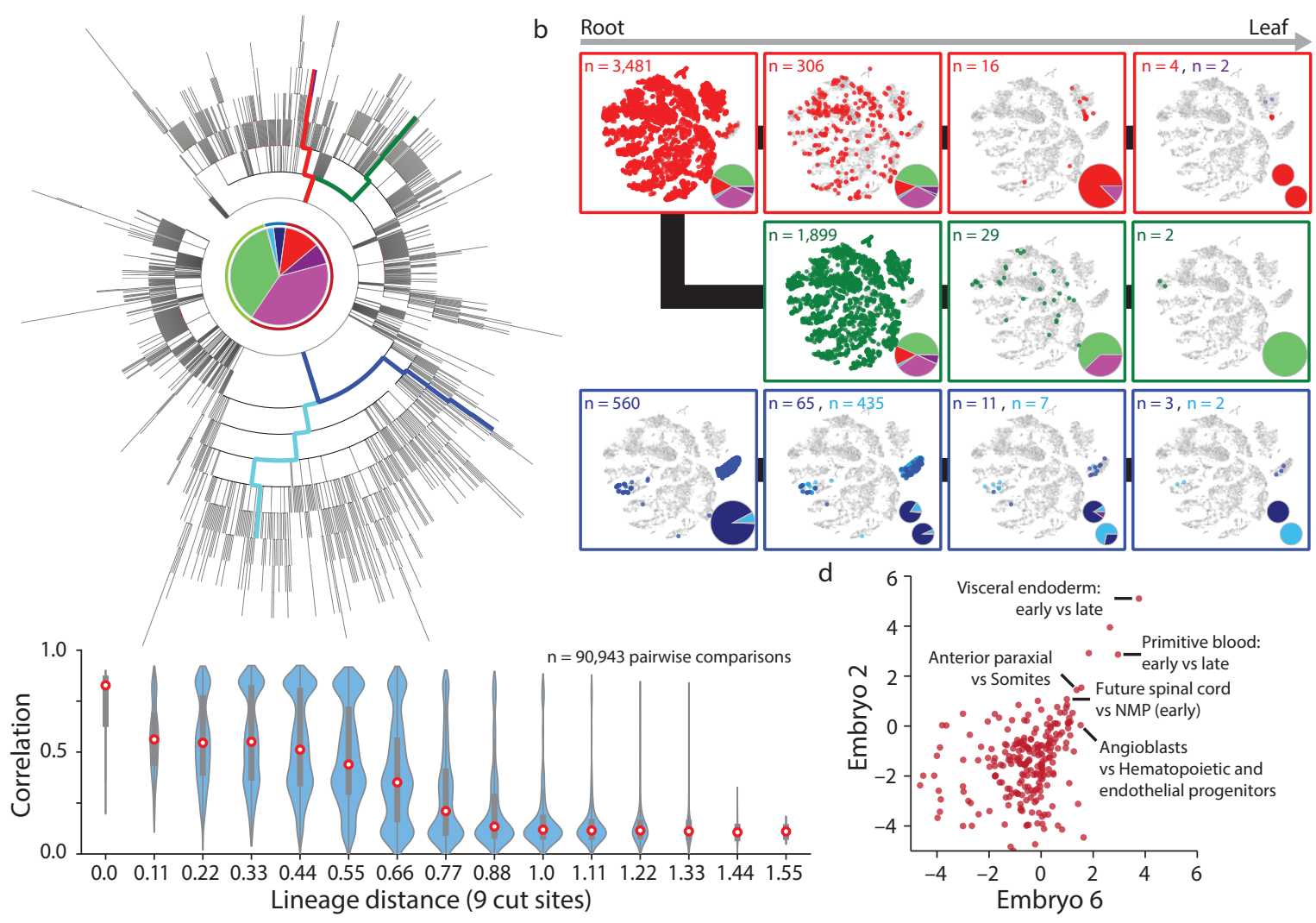



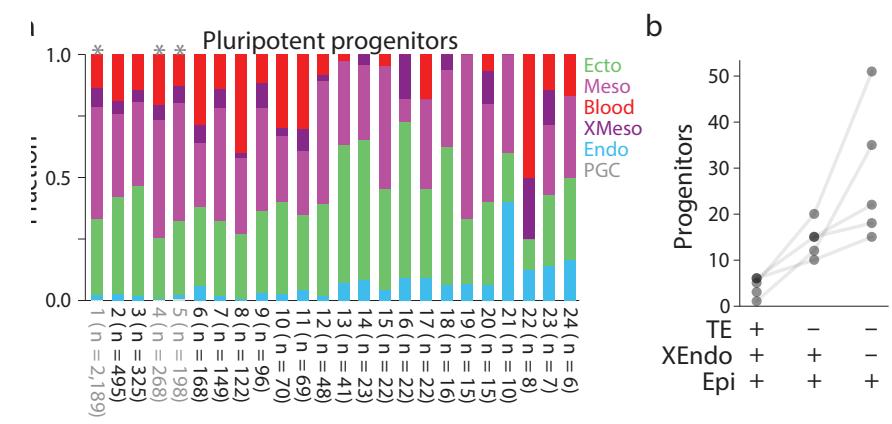